\title{
Concerning continuous curves ${ }^{1}$ ).
}

By

\author{
R. L. Wilder (Austin, U. S. A.).
}

It has been shown' by $\mathrm{Hahn}{ }^{2}$ ), and independently by Mazurkiewiczs) that in order that a closed, bounded and connected ") point-set should be a continuous curve (une ligne de Jordan) it is necessary and sufficient that it be connected im kleinen at every point. A point-set $M$ is connected im kleinen at a point $P$ if tor every $\varepsilon>0$ there corresponds an $\eta>0$, such that if $K_{1}$ and $K_{2}$ are circles with radii $\varepsilon$ and $\eta$, respectively, and centers at $P$, every point $X$ of $M$ interior to $K_{2}$ lies with $P$ in a cónnected s) subset of $M$ that lies wholly interior to $K_{1}$. A point-set that is connected im kleinen at every point is said to be connected im kleinen.

The present paper has three main objects, viz.,

(1) To study the analogy between ordinary two-dimensional space and a plane continuous curve,

(2) To characterize and analyze the boundaries of the domains complementary to a plane continuous curve, and

1) Dissertation offered to the Department of Pure Mathematics, University of Texas, U. S. A., in partial fulfilment of the requirements for the degree of Doctor of Philosophy, June, 1923.

1) Mengentheoretische Charaiterisierung der stetigen Kurve, Wiener Akademie Sitzungsberichte, CXXIII Band. Abt. IIa, pp. 2433-2489.

3) Sur les lignes de Jordan, Fund. Math., Tom I, (1920), pp. 166-209.

1) A point-set is said to be connected if it is not the sum of two point-sets neither of which contains a limit point of the other. A point-set is bounded if it lies wholly in a finite portion of the space under consideration.

5) According to Hahn's definition $X$ and $P$ must lio together in a closed and connected subset of $M$ that lies wholly interior to $K_{1}$. The word ${ }_{n}$ closed $^{\mu}$ is unnecessary, however; when dealing with closed point sets. 
(3) To give a new characterization of continuous curves, suitable for any number of dimensions.

I wish to thank Professor R. L. Moore for many valuable suggestions and criticisms, and to express my gratitade to him for first interesting $m e$ in a field of mathematics that is as fascinating as it is fruitful.

\section{A continuous curve in the role of a. space ${ }^{1)}$.}

For the present, I shall consider a space. $S$, which consists of all the points of a plane continuous curve. I shall define a region in that space as follows: If $P$ is a point of $S$, and $k$ a circle with center at $P$, then the set of all points of $S$ which (1) lie interior to $k$, and (2) lie with $P$ in a connected subset of $S$ that lies wholly interior to $k$, constitutes a region $R$. A point $P$ is a limit point of a point-set $M$ in space $S$ if and only if every region that contains $P$ contains at least one point of $M$ distinct from $P$. This definition of limit point is equivalent to the ordinary definition of limit point for two-dimensional space, in that a point $P$ which is a limit point of a point-set $M$ in space $S$ is also a limit point of $M$ in the ordinary sense, and vice versa. The set of all limit points of $R$ that do not belong to $R$ constitute the boundary of $R$. Every boundary point of $R$ lies on $k$. A domain with respect to $S^{2}$ ), or an $S$-domain is a connected subset $D$ of $S$ having the property that if $P$ is a point of $D, P$ lies in some region that is a subset of $D$. The set of points, that are limit points of $D$ but that do not belong to $D$, constitute the boundary of $D$.

Using the above definitions, it is interesting to note that many of the properties of ordinary two-dimensional space are also properties of space $S$. In particular, Theorems 1-16, inclusive, (with the, exception of the latter part of Theorem 16) of R. L. Moore's paper On the foundations of plane analysis situs s) all hold true for space $S$ with no change in the wording, except that the word "domain " should be replaced by ${ }_{n} S$-domain".

1) Presented to the American Mathematical Society, in part, April 15, 1922 , and in more complete and generalized form, Feb. 21, 1923.

. 3) Kuratowski has used an analogous definition for what he calls domaino: connexe par rapport a $C \star$. Cf. C. Kuratowsi, Une definition topologique de la ligne de Jordan. Fund. Math. I, (1920), pp. 40-43.

3) Transactions of the Amer. Math. Soc., Vol. XVII, (1916), pp. 131-164. 
Two of these theorems which are of fundamental importance are:

If $A$ and $B^{a}$ are distinct points of a domain $M$, there exists a simple continuous. arc ${ }^{1}$ ) from $A$ to $B$ that lies wholly in $M$.

Every two points of a region $R$ can be joined by an are lying entirely in $R$.

The close analogy between space $S$ and ordinary two-dimensional space can be further exhibited by a consideration of accessibility conditions 2). For this purpose, I shall establish the following theorem:

Theorem 1. In order that a boundary point $x$ of an $S$-domain $D$ should be accessible from any point $y$ of $D$, it is sufficient either that (1) there exist a circle $C_{1}$ with center at $x$, such that the set of all points of the boundary, $B$, that lie interior to $C_{1}$ is a subset of a connected im kleinen subset of $S-D$, or that (2) $x$ belong to no. continuum of condensation ${ }^{3}$ ) of $B$.

Proof. I shall consider these two conditions separately.

(1) There exists a circle $C_{1}$ with center at $x$, such that if $L$ is the set of all points of $B$ that lie within $C_{1}, L$ is a subset of a connected im kleinen subset $K$ of $S-D$. The circle $C_{1}$ may be taken so small that it does not enclose $y$ or contain $y$.

Since $x$ is a boundary point of $D$, and therefore a limit point of $D$, there exists in $D$ a sequence of distinct points $y_{1}, y_{2}, y_{3}, \ldots$ having $x$ as a sequential limit point $\left.{ }^{4}\right) . C_{1}$ encloses some polnt of this sequence. Call one such point $P_{1}$. Let $C_{2}$ be a circle concentric with $C_{1}$, of radius $<r / 2$, (where $r$ is the radius of $C_{1}$ ) and such that $P_{1}$ is exterior to $C_{2}$. $C_{2}$ encloses some point of the above

1) A simple continuous arc, or an arc, from $A$ to $B$ is a closed, bounded and connected set of points that is disconnected by the omission of any one point except $A$ and $B . A$ and $B$ are called the end.points, or extremities, of this arc, and all other points of the arc are interior points. Two points are said to be joined by an are if they are the end-points of that arc.

2) If $x$ is a boundary point of a domain in the ordinary sense, or an $S$-domain) $D$, and $y$ a point of $D, x$ is said to be accessible from $y$ provided there exists an arc from $x$ to $y^{\prime}$ which lies, except for $x$, wholly in $D$.

s) A continuum is a closed und connected point-set consisting of more than one point, A point-set $C$ is a continuum of condensation of a point-set $M$ if $C$ is a sub-continnum of $M$ such that every point of $C$ is a limit point of $M-C$.

4) A point $P$ is a sequential limit point of a sequence of points $P_{1}, P_{2}, P_{3}, \ldots$ provided that if $R$ is a region containing $P$ there exists a number $N$ such that if $n>N, P_{n}$ lies in $R$. 
sequence; call one such point $P_{2}$. Let $C_{8}$ be a circle concentric with $C_{2}$, of radius $<r / 3$, and such that $P_{2}$ is exterior to it. $C_{3}$ encloses a point $P_{8}$ of the above sequence. Continuing this process indefinitely, there is obtained a sequence of circles, $C_{1}, C_{2}, C_{2} \ldots$ with centers at $x$ and such that for every positive integer $n>1$, $C_{n}$ is of radius $\left\langle r / n\right.$; and a sequence of points, $P_{1}, P_{2}, P_{3}, \ldots$ belonging to $D$, such that for every positive integer $n, P_{n}$ lies interior to $C_{n}$, but exterior to $C_{n+1}$.

Since, as pointed out above, any two points of $D$ are the extremities of an are lying wholly in $D, y$ and $P_{1}$ are the extremities of an arc $a_{1}, P_{1}$ and $P_{2}$ of an arc $t_{2}, P_{2}$ and $P_{3}$ of an arc $t_{3}$, etc., such that $a_{1}, t_{2}, t_{3}, t_{4} \ldots$ all lie wholly in $D$. Let $x_{2}$ be the last point of $a_{1}$ on the arc from $P_{1}$ to $P_{3}$, in the order from $P_{1}$ to $P_{2}$. (See Fig. 1). That portion of $t_{2}$ from $x_{2}$ to $P_{2}$ constitutes an arc $a_{2}$ Since $a_{1}+a_{8}$ is a closed set of points, there will be a last point of it on the arc $t_{8}$ in the order from $P_{2}$ to $P_{8}$; call this point $x_{9}$. That portion of $t_{3}$ from $x_{3}$ to $P_{3}$ constitutes an arc $a_{3}$. The set $a_{1}+$ $+a_{2}+a_{8}$ is closed, and there therefore exists a last point of it, $x_{4}$, on the arc $t_{4}$ in the order from $P_{3}$ to $P_{4}$. That portion of $t_{4}$ from.

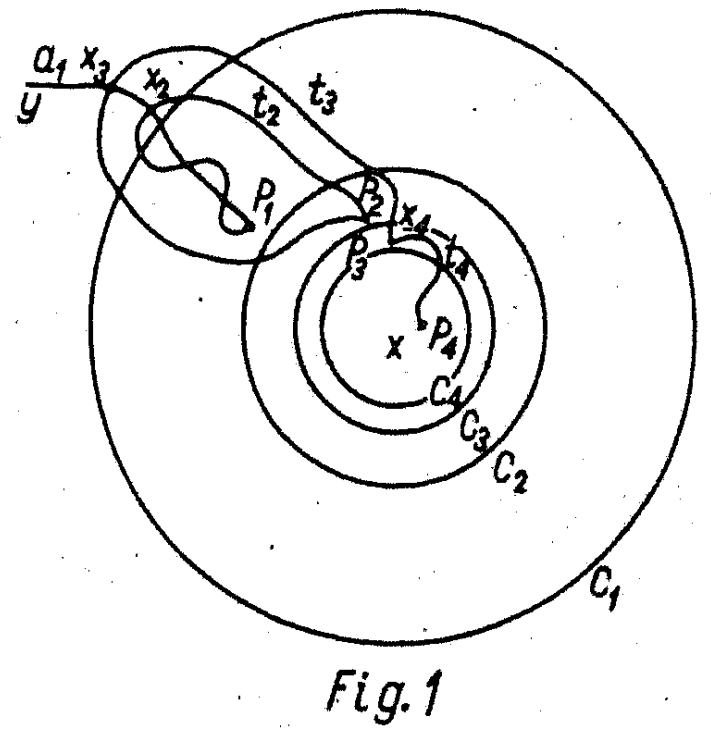
$x_{4}$ to $P_{4}$ constitutes an arc $a_{4} \ldots$. Continuing this process indefinitely, there is obtained a sequence of arcs, $a_{1}, a_{2}, a_{3}, \ldots$ such that for every positive integer $n, a_{n}$ has only one point, an end-point $x_{n}$, in common with the set $a_{1}+a_{2}+a_{3}+\ldots+a_{n-1}$.

There are two cases to consider: Either (1) for every value of $n$ there, exists a positive integer $k$, such that $a_{k}$ is the last are of the sequence $a_{1}, a_{2}, a_{3}, \ldots$ having points on $C_{n}$; or (2) there exists an $n$, such that an infinite number of ares of the sequence $a_{13}, a_{3}, a_{3}, \ldots$ have points on $C_{n}$.

Case 1. For every value of $n$ there exists a positive integer $k$, such that $a_{k}$ is the last are of the sequence $\left[a_{i}\right]^{\text {i) }}$ ) having points

1) Hereafter, in the proof of this theorem, I shall denote the sequence $a_{1}$ $a_{2}, a_{3}, \ldots$ by the symbol $\left[a_{i}\right]$. 
on $C_{n}$. Then the point-set constituted by all the arcs of the sequence. $\left[a_{i}\right]$ together with the point $x$ is a continuous curve and contains an arc from $x$ to $y^{1}$ ) every point of which, except $x$, belongs to $D$. In this case, then, the theorem is true.

Case 2. There exists an $n$, such that an infinite number of the arcs of $\left[a_{i}\right]$ have points on $C_{n}$. It is certain that an infinite number of arcs of $\left[a_{i}\right]$ have points exterior to $C_{n+1}$, and points interior to $C_{n+2}$. For every such arc, $a_{i}$, let $A_{i}$ be the first point of $C_{n+1}$ on $a_{i}$ in the order from $P_{i}$ to $x_{i}$. (See Fig. 2). Then, let $B_{i}$ be the first point of $C_{n+2}$ on that portion of $a_{i}$ from $A_{i}$ to $P_{i}$, in the order

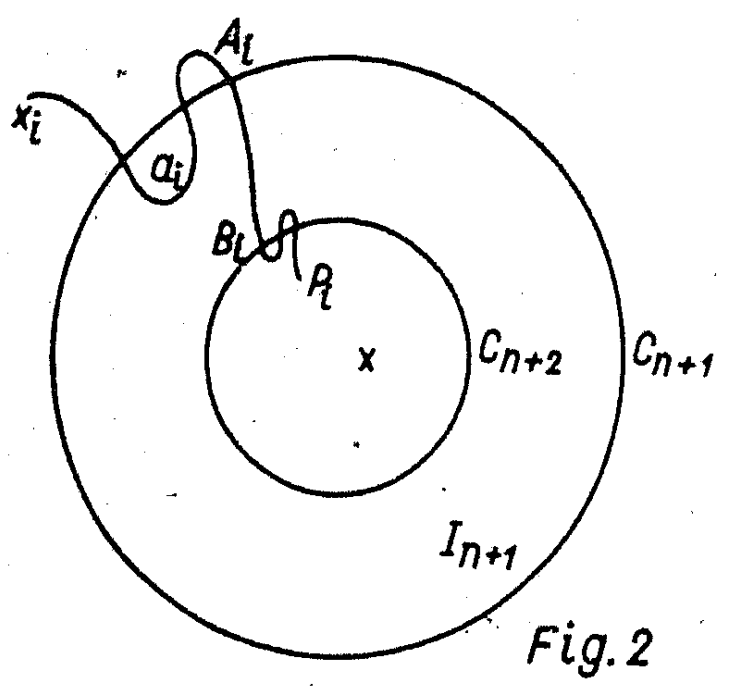
from $A_{i}$ to $P_{i}$. Then from $A_{i}$ to $B_{1}$ there exists an arc which is a subset of $a_{i}$, and such that if $I_{n+1}$ is the set of all points of the plane between $C_{n+1}$ and $C_{n+2}, A_{i} B_{i}$ is a subset of $I_{n+1}$ except for the points $A_{i}$ and $B_{i}$. The set of all such arcs call $\left[a_{i}^{*}\right]$.

No two arcs of the set $\left[a_{i}^{*}\right]$ have any points in common. For, suppose $A_{k} B_{k}$ and $A_{m} B_{m}$ are two arcs of the set $\left[a_{i}^{*}\right]$ having a point, $x^{*}$, in common, and that $k<m$. Then $a_{m}$ was taken subsequently to $a_{k}$, and can therefore have at most one end-point, $x_{m}$, in common with $a_{k}$. Other than $x_{m}, a_{k}$ and $a_{m}$ can have no points in common. Hence $x^{*}$ must be identical with $x_{m}$. But $A_{m}$ is the first point of $C_{n+1}$ on $a_{m}$ in the order from $P_{m}$ to $x_{m}$, and $A_{m} B_{m}$ is therefore a subset of that portion of $a_{m}$ from $P_{m}$ to $A_{m}$, and unless $A_{m}$ is identical with $x_{m}, A_{m} B_{m}$ can have no point in common with $A_{k} B_{k}$. But if $A_{m}$ is identical with $x_{m}, a_{m}$ can have no points exterior to $C_{n+1}$, which is contradictory, since

1) Every two points of a continuous curre $M$ can be joined by a simple continuous are which is a sabset of $M$. For a proof of this, see R. L. Moore, 4 theorem concerning continuous curves, Bull. Amer. Math. Soc. 2d. series, XXIII (Feb. 1917), S. 233-236. See also R. Tietze, Uber stetiye Kurven, Jordansche Kurvenbogen und geschlossene Jordansche Kurven, Math. Zeitschr. V (1919), 8. 284-291; and. S. Mazurkiewicz, Sur les lignes de Jordan, loc. cit. In this article, Mazurkiewicz establishes numerons resalts and indicates that some of them were published in a journal (C. R. Soc. Sc. Varsovie) to which, I have not had access. 
$a_{m}$ was taken as one of the ares of $\left[a_{i}\right]$ having points exterior to $C_{n+1}$. Hence $A_{m} B_{m}$ and $A_{k} B_{k}$ have no point in common.

The infinite set of points of the type $A_{1}$ has at least one limit point on $C_{n+1}$. Let $\bar{A}$.be one such point. If $z$ is any other point on $C_{n+1}$, then at least one of the arcs into which $\bar{A}$ and $z$ divide $C_{n+1}$ must contain an infinite set of points of the type $A_{i}$ having $\bar{A}$ as a limit point; call this are $\bar{A} z$. Then from the points of the type $A_{i}$ can be selected (See Fig. 3 ) an infinite sequence $\bar{A}_{1}, \bar{A}_{2}, \bar{A}_{3}, \ldots$ in the order from $z$ to $\bar{A}$ on $\bar{A} z$, and having $\bar{A}$ as a sequential limit point. The set of points $\bar{B}_{1}, \bar{B}_{2}, \bar{B}_{8}, \ldots$ where $\bar{B}_{n}$ is the other end-point of the are of $\left[a_{i}^{*}\right]$ to which $\bar{A}_{n}$ belongs has a sequential limit point $\bar{B}$. There is obtained thus a sequence $\overline{A_{1}} \bar{B}_{1}, \overline{A_{2}} \overline{B_{2}}$, $\overline{A_{3}} \bar{B}_{3}, \ldots$ of arcs of the set $\left[a_{i}^{*}\right]$ arranged in a definite order. Call this sequence the set $\left[\overline{A_{i}} \overline{B_{i}}\right]$.

The set $\left[\overline{A_{i}} \overline{B_{i}}\right]$ has a closed and connected limiting set $\left.{ }^{1}\right), M_{1}$, which has at least one point $(\bar{A})$

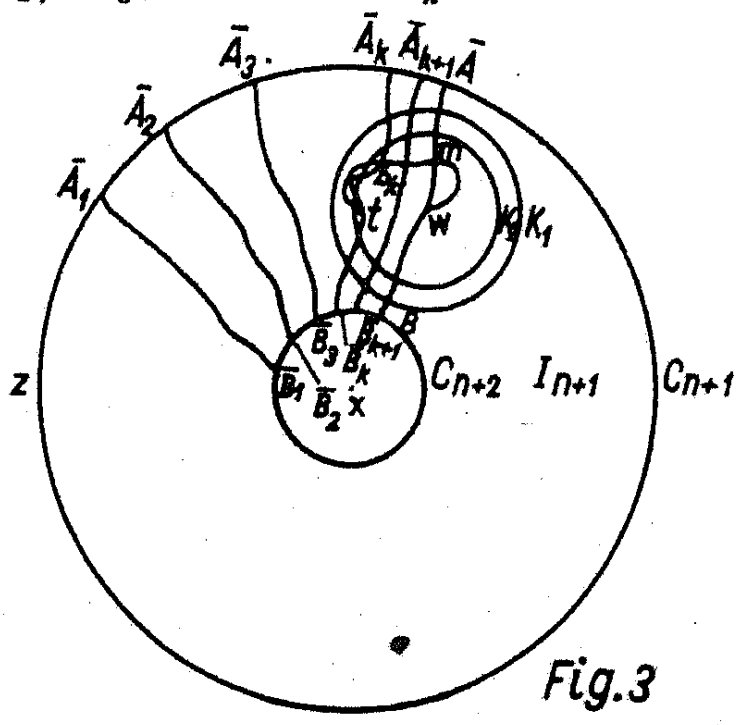
on $C_{n+1}$, and at least one point $(\bar{B})$ on $C_{n+2}$. Let $w$ be a point of $M_{1}$ within $I_{n+1}$, and let $K_{1}$ be a circle with center $w$ and lying wholly in $I_{n+1}$, but not enclosing $C_{n+2}$. Becanse of the property of connectivity im kleinen of $S$, there exists, concentric with, and lying interior to $K_{1}$, a circle $K_{2}$, such that every point of $S$ interior to $K_{\mathrm{q}}$ is joined to $w$ by an arc ${ }^{2}$ ) of $S$ lying wholly interior to $K_{1}$. Let $\bar{A}_{k} \bar{B}_{k}$ be the first are of the set $\left[\bar{A}_{i} \bar{B}_{i}\right]$ having points interior to $K_{\mathbf{2}}$.

It is certain that no point on any are $\bar{A}_{n} \bar{B}_{n}$ can be joined to $w$ by an are lying wholly interior to $K_{1}$ unless this arc contains points of all arcs of the set $\left[\overline{A_{i}}, \overline{B_{i}}\right]$ of subseript $>\boldsymbol{n}$.

1) Cf. B. Janiszewski, Sur los continus irréductibles entre deux points, Journal de l'Ecole Polytechnique, (2), XVI, (1912), p. 109. Th. 1.

2) It is permissible to say, arce here instead of sconnected set since all points of $S$ lying interior to $K_{2}$ belong to that region of $S$ determined by $K_{1}$, and since every two points a a region are the extremities of an arc lying wholly in that region, 
There are two casses:

(a) If $w$ is a point of $D, K_{1}$ can be taken so small as to contain no points of $S-D$, in which event all ares of the set $\left[\bar{A}_{i} \bar{B}_{i}\right]$ of subseript $\geqq K$ are connected by an arc of $D$ lying wholly interior to $K_{1}$.

(b) If $w$ is a point of $B$ :

For each $n, \bar{A}_{n} \bar{B}_{n}$ and $\bar{A}_{n+1} \bar{B}_{n+1}$, together with those arcs $\bar{A}_{n} \bar{A}_{n+1}$ and $\bar{B}_{n} \bar{B}_{n+1}$ of $C_{n+1}$ and $C_{n+2}$, respectively, that contain no end-points of the set $\left[\bar{A}_{i} \overline{B_{i}}\right]$, form a simple closed curve $\bar{K}_{n}$ which cannot enclose $w$. Let $t$ be a point of $\bar{A}_{k} \bar{B}_{k}$ interior to $K_{\mathrm{g}}$. An arc of $S$ from $t$ to $w$ lying wholly interior to $K_{1}$, must contain, for every $\bar{K}_{n}$ (where $n \geqq k$ ) an are $b_{n}$. such that $b_{n}$ lies wholly interior to $\bar{K}_{n}$ except for its end-points; but for each $\bar{K}_{n}$ there can be at most a finite number of arcs' such as $b_{n}$. If $w$ is not the first point of $M$, on the are from $t$ to $w$, in the order from $t$ to $w$, let $m$ be that first point. Let $\left[b_{n}\right]$ desote the set of all ares of the type $b_{n}$ on the arc from $t$ to $m$.

If only a finite number of ares of $\left[b_{n}\right]$ contain points of $S-D$, then there exists an $n(n \geqq k)$ such that if $i>n, \bar{A}_{i} \overline{B_{i}}$ and $\overline{A_{i+1}} \bar{B}_{i+1}$, are connected by an are of $D$ lying wholly interior to $K_{1}$.

I shall show next that it is impossible that an infinite number of the ares $\left[b_{n}\right]$ contain points of $S-D$.

If any arc, $b_{n}$, contains points of $S-D$, it must, since its endpoints are in $D$, contain points of $B$ So, if an infinite number of arcs of $\left[b_{n}\right]$ contain points of $S-D$, the same arcs contain points of $B$; that is, every such $b_{n}$ contains at least one point, $d_{m}$, of $B$. Call the set of such points $\left[d_{n}\right]$.

Clearly, $m$ is the limit point in $M_{1}$ of the set of end-points of the set $\left[b_{n}\right]$. For the end-points belong to the set $\left[\bar{A}_{i} \overline{B_{i}}\right]$, so that their limit point must lie in $M_{1}$, and if any other point, $m^{\prime}$, of $M_{1}$, were this limit point. it would mean that a set of points in the are from $t$ to $m$ has a limit point exterior to the are ( $m$ is the first point of $M_{1}$ on the arc), an impossibility, since an are is a closed set. But if $m$ is the limit point of the end-poinls of the set $\left[b_{n}\right]$ it is the limit point of the end points of those ares to which the set $\left[d_{n}\right]$ belongs, and hence also the limit point of the set $\left[d_{n}\right]$. There- 
fore $m$ must belong to $L$ since $B$ is a closed set. But $L$ is a subset of $K$; thus $m$ is a point of $K$ and the set $\left[d_{n}\right]$ belongs to $K$.

Let $K_{1}^{*}$ be a circle with center at $m$ and lying in $I_{n+1}$, but not enclosing $C_{n+2}$. Since $K$ is connected im kleinen, there exists, concentric with, and lying interior to $K_{1}^{*}$, a circle $K_{2}^{*}$, such that all points of $K$ lying interior to $K_{2}^{*}$ can be joined to $m$ by a connected subset of $K$ lying interior to $K_{1}^{*}$. But $K_{2}^{*}$ must enclose points of $\left[d_{n}\right]$, and a connected set containing a point of $\left[d_{n}\right]$ and $m$ and lying interior to $K_{1}^{*}$ must contain points of $D$; that is, points not belonging to $K$. Hence the contradiction:

In any case, then, there must exist a number $\delta_{1}$, such that for. $i>\delta_{1}$ and $j>\delta_{1}$, any are $\bar{A}_{i} \bar{B}_{i}$ is connected to any other arc $\overline{A_{j}} \overline{B_{j}}$ by an arc of $D$ lying entirely interior to $K_{1}$. This means, then, that those arcs of the set $\left[a_{i}\right]$ of which the arcs of $\left[\bar{A}_{i} \bar{B}_{i}\right]$ of subscript $>\delta_{1}$ are subsets, can be joined by arcs of $D$ in the same manner. Call the set of these ares $S_{1}$.

For each are $a_{i}$ of $S_{1}$, that contains points interior to $C_{n+3}$ let $D_{i}$ be the last point of $C_{n+2}$ on $a_{i}$ in the order from $x_{i}$ to $P_{i}$ and $E_{i}$ the first point of $C_{n+8}$ on that portion of $a_{i}$ from $D_{i}$ to $P_{i}$, in the order from $D_{i}$ to $P_{i}$. Then each such $a_{i}$ contains an are $D_{i} E_{i}$, such that $D_{i} E_{i}$ lies wholly in $I_{n+2}$ (where $I_{n+2}$ is the set of all points of the plante between $C_{n+2}$ and $\left.C_{n+8}\right)$ except for its end-points. The set of all such arcs eall $\left[D_{i} E_{i}\right]$ It ean be proved, by the method used above, that the arcs of the set $\left[D_{i} E_{i}\right]$ can be ordered in a sequence $\bar{D}_{1} \bar{E}_{1}: \bar{D}_{2} \bar{E}_{2}, \bar{D}_{8} \bar{E}_{8}, \ldots$ having the property that there exists a $\delta_{2}$, such that for $i>\delta_{2}$ and $j>\delta_{2}$, any arc $\bar{D}_{1} \bar{E}_{1}$ is connected to any other arc $\bar{D}_{j} \bar{E}_{j}$ by an arc of $D$ lying wholly in $I_{n+2}$. Those arcs of $S_{1}$ which contain arcs of $\left[\bar{D}_{i} \overline{E_{i}}\right]$ of subscript $>\delta_{2}$ call $S_{2}$.

This process may be repeated indefinitely, since $S_{2}$ contains an infinite number of arcs of the set. $\left[a_{i}\right]$ and thereafter any $S_{i}$ will have the same property. Furthermore, for any $j,(1) S_{j}$ will be a subset of $S_{j-1}^{\prime},(2)$ if $a_{k}$ and $a_{m}$ are any two ares of $\left[a_{i}\right]$ which belong to $S_{j}$, there exists an arc $a b$ or $D$ such that (i) one end-point of this arc, $a$, is a point of $a_{k}$, and the other end-point, $b$, is a point of $a_{m}$, (ii) the arc $a b$ lies wholly interior to some circle. which lies in $I_{n+s}$ (the annular domain bounded by $C_{n+3}$ and $C_{n+j+1}$ ). but which does not'enclose $C_{m+s+1}$, and (iii) the ares $a P_{k}$ and $b P_{m}$; subsets of $a_{k}$ and $a_{m}$, respectively, lie wholly interior to $C_{n+j}$ 
The first arc of the sequence $\left[a_{i}\right]$ belonging to $S_{1}$ call $c_{1}$. The first are of $\left[a_{i}\right]$ after $c_{1}$ which belongs to $S_{2}$ call $c_{2}$. Then there exists, interior to $K_{1}$, an arc of $D$ from a point $x^{\prime}$ on $c_{1}$ to a point $y^{\prime}$ on $c_{2}$, and such that the arc $y^{\prime} P_{t}$, a subset of the arc $c_{2} \equiv a_{t}$, lies wholly interior to $C_{n+1}$ (See Fig. 4). The first arc of $\left[a_{i}\right]$ after $c_{2}$ which belongs to $S_{3}$ call $c_{3}$. Then there exists, interior to $I_{n+2}$, an arc of $D$. from a point $x^{\prime \prime}$ of the $\operatorname{arc} y^{\prime} P_{t}$ to a point $y^{\prime \prime}$ of the

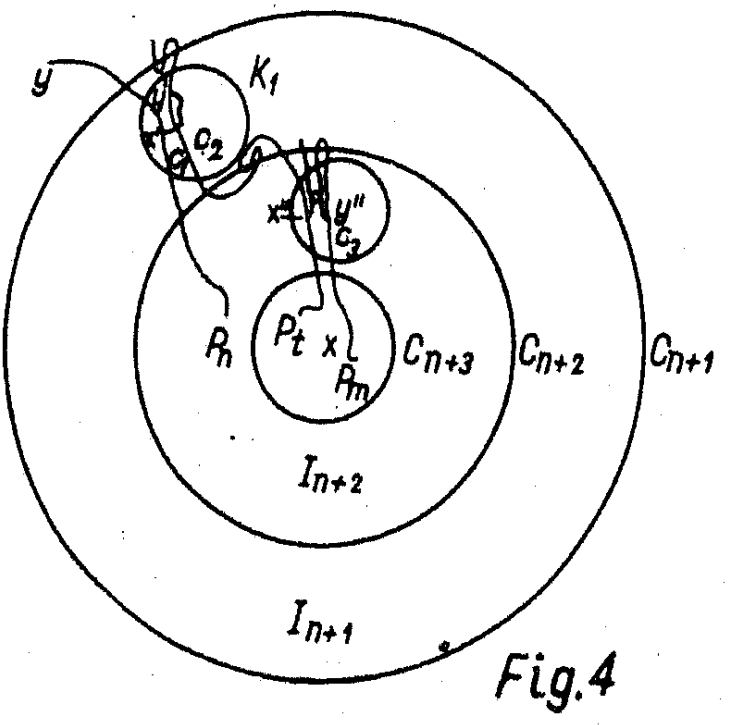
arc $c_{g}$, and such that the arc $y^{\prime \prime} P_{m}$ (where $c_{3} \equiv a_{m}$ ) subset of $c_{3}$, lies wholly interior to $C_{n+2}$. Continue this process indefinitely. Then, if $a_{k} \equiv c_{1}$, the set $a_{1}+a_{2}+\ldots .+a_{k}+$ $+x^{\prime} y^{\prime}+y^{\prime} P_{t}+x^{\prime \prime} y^{\prime \prime}+y^{\prime \prime} P_{m}+$ $+\ldots+x$ can be shown, as in Case 1, to contain an arc from $x$ to $y$, every point of which, except $x$, is a point of $D$. 'This completes. the proof for Case 2.

(2) $x$ belongs to no continuum

of condensation of $B$.

Choose the sequence of circles $C_{1}, C_{2}, C_{8}, \ldots$, the sequence of points $P_{1}, P_{2}, P_{3}, \ldots$ and the sequence of arcs $a_{1}, a_{2}, a_{2}, \ldots$ as in (1) starting, however, with $C_{1}$ as any circle with center at $x$. As in (1), there are two cases to consider:

Case I. Suppose that for every value of $n$ there exists a positive integer $k$, such that $a_{k}$ is the last are of the sequence $a_{1}, a_{2}, a_{3} \ldots$ having points on $C_{n}$. This situation is handled exactly as in Case 1 of (1).

Case 2. Suppose there exists an $n$, such that an infinite number of arcs of $\left[a_{i}\right]$ have points on $C_{n}$. Select the set $\left[a_{i}^{*}\right]$ as in Case 2 of (1), with the difference that to each $A_{k} B_{k}$ has been added the arc $B_{k} P_{k}$ forming the arc $A_{k} P_{k} \equiv a_{k}^{*}$. As above, it can be shown that no two ares of $\left[a_{i}^{*}\right]$ have puints in common.

The points of the type $A_{1}$ have at least one limit point $\bar{A}$ on $C_{n+1}$. Let $z$ be any point of $C_{n+1}$ distinct from $\bar{A}$. Then at least one of the arcs into which $z$ and $\bar{A}$ divide $C_{n+1}$ contains an infinite number of points of the type $A_{i}$ call this arc $\bar{A} z$. Choose on $\bar{A} z$, in the order from $z$ to $\bar{A}$, an infinite sequence of points of type $A_{i}$, 
namely. $A_{1}^{*}, A_{2}^{*}, A_{3}^{*}, \ldots$ having $\bar{A}$ as a sequential limit point. The set of points, $P_{1,}^{*}, P_{2}^{*}, P_{3}^{*}, \ldots$, where for every positive integer $n, P_{n}^{*}$ is the other end-point of the arc of $\left[a_{i}^{*}\right]$ to which $A_{n}^{*}$ belongs, has $x$ as a sequential limit point, since $x$ is a sequential limit point of the set $P_{1}, P_{2}, P_{3}, \ldots$ The sequence of arcs $A_{1}^{*} P_{1}^{*}, A_{2}^{*} P_{2}^{*}, A_{8}^{*} P_{3}^{*}, \ldots$ has a continuurn $M_{2}$ as a sequential limiting set; $M_{2}$ contains $\bar{A}$ and $x$

Either there exists in $M_{2}$ an infinite set of points of $D$ having $x$ as a sequential limit point, in which event a proof similar to that used in Case 2 (a) of (1) can be used to show the accessibility of $x$ from $y$, or there exists no such sequence. In the latter event we can consider $C_{n+1}$ to be so small that all points of $M_{2}$ belong to $S-D$. Then $M_{2}$ is a subset of $B$, since each point of it is a limit point of $D$ and does not belong to $D$.

Let $e_{1}$ be any point of $M_{2}$ interior to $C_{n+1}$ and distinct from $x$. Let $K_{1}^{\prime}$ be a circle with $e_{1}$ as center, not enclosing $x$ or containing $x$, and lying entirely interior to $C_{n+1} . K_{1}^{\prime}$ determines a concentric circle $K_{2}^{\prime}$ such that every point of $S$ interior to $K_{2}^{\prime}$ is joined to $e_{1}$ by an are of $S$ that lies wholly interior to $\bar{K}_{1}^{\prime}$. There exists a positive number $N$ such that for $n>N, K_{2}^{\prime}$ cuts off at least one segment of $A_{n}^{*} P_{n}^{*}$. Let $t$ be a point of $A_{E}^{*} P_{\Gamma}^{*}(K>N)$ interior to $K_{\triangleright}^{*}$ and let $m$ be the first point of $M_{2}$ on an are of $S$ from $t$ to $e_{1}$ that lies entirely interior to $K_{1}^{\prime}$ (in the order from $t$ to $e_{1}$ ). For every $n>N$ let $p_{n} q_{n}$ and $p_{n+1} q_{n+1}$ be arcs" of $A_{n}^{*} P_{n}^{*}$ and $A_{n+1}^{*} P_{*+1}^{*}$, respectively, cut off by $K_{1}^{\prime}$ (i. e. $p_{n} q_{n}$ lies entirely interior to $K_{1}^{\prime}$ except for the points $p_{\mathrm{n}}$ and $q_{\mathrm{n}}$, etc.) and such that one of the arcs into which $p_{n}$ and $p_{n+1}$ divide $K_{1}^{\prime}$ does not contain $q_{n}$ or $q_{n+1}$. Let $p_{n} q_{n+1}$ be that simple closed curve formed by the arcs $p_{n} q_{n}, p_{n+1}$ $q_{n+1}$, that arc $p_{n} p_{n+1}$ of $K_{1}^{\prime}$ which contains neither $q_{n}$ nor $q_{n+1}$, and that arc $q_{n} q_{n+1}$ of $K_{1}^{\prime}$ which contains neither $p_{n}$ nor $p_{n+1}$.

Two possible situations have to be considered. I shall say that $e_{1}$ possesses property $F$ if, no matter how $K_{1}^{\prime}$ is seleeted, subject to the conditions noted above, or how $t m$ is selected, the are $t m$ contains, tor an infinite number of distinct values of $n$, a point of $S-D$ that lies interior to $p_{n} q_{n+1}$. Either $e_{1}$ possesses property $F$ or it does not; in the latter event, $e_{1}$ will be said to possess property $G$.

If $e_{1}$ possesses property $G$, let $C_{n+2}^{*}$ be a circle concentric with $C_{n+1}$, not enclosing $e_{1}$ and lying interior to $C_{n+2}$. There exists 
a subcontinuum $M_{8}$ of $M_{2}$, which contains $x$ and at least one point on $C_{n+2}^{*}$, but no points exterior to $C_{n+2}^{*}$. Either a point $e_{2}$ of $M_{8}$ interior to $C_{n+2}^{*}$ and distinct from $x$ can be found possessing property $G$ or such a point cannot be found. If such a point ean be found, let $C_{n+3}^{*}$ be a circle concentric with $C_{n+2}^{*}$, not enclosing $e_{2}$, and lying interior to $C_{n+8}$. There exists a subcontinuum $M_{4}$ of $M_{3}$ which contains $x$ and at least one point on $C_{n+s}^{*}$, but no points exterior to $C_{n+3}^{*}$. A point $e_{3}$ of $M_{4}$ interior to $C_{n+3}^{*}$ and distinct from $x$ can be found possessing property $G$, or no such point can be found. If such a point can be found, continue as before. In the event that this process can be kept up indefinitely, that is, if for every new $C_{n+i}^{*}$ lying within $C_{n+i}$ a point $e_{i}$ interior to $C_{n+i}^{*}$ can be found possessing property $G$, then there exists a circle $K_{1}^{i}$ with center at $e_{i}$ and lying within $C_{n+i}^{*}$ such that an infinite number of ares of $\left[a_{i}^{*}\right]$ that have points interior to $K_{1}^{i}$ can be connected by an are. of $D$ lying wholly interior to $K_{1}^{i}$. The method used in Case $2(b)$ of (1) can' now be used to show the accessibility of $x$.

In the event that this process can not be kept up indefinitely; that is, if finally a circle $C_{n+i}^{*}$ is found such that every point of $M_{i}$ interior to $C_{n+i}^{*}$ possesses property $F$, we may proceed as follows: Consider $C_{n+1}$ taken so that every point of $M_{2}$ other than $x$ interior to $C_{n+1}$ possesses property $F$. But then every point of $M_{2}$ other than $x$ is a limit point of boundary points of $B$ that do not belong to $M_{9}$, and sinee $M_{2}$ is a continuum, $x$ itself is a limit point of such points. That is, $x$ belongs to a continurm of condensation, $M_{2}$, of $B$. contrary to hypothesis.

Hence, in every case, $x$ is accessible from $y$ if it belongs to no continuum of condensation of the boundary.

This completes the proof of Theorem 1.

It will be noticed that in the above proof no properties of space $S$ are made use of, that are not also properties of ordinary two-dimensional space. The arguments used. apply, therefore, in the latter space. Hence the following theorem:

Theorem 2. In order that a boundary point $x$ of a connected domain $D$ in ordinary two-dimensional space should be accessible from all points of $D$, it is sufficient either that (1) there exist a circle $C$ with centre at $x$ such that the set of all points of the boundary, $B$, interior to $C$ is a subset of a connected im kileinen subset of $S-D$ (S being 
the set of all points in the space considered), or that (2) $x$ belong to no continuum of condensation of $B{ }^{1}$ ).

It is clear that the conditions of Theorem 2 are satisfied if any one of the following simpler conditions are satisfied, viz., that (a) $B$ be connected im kleinen, (b) $B$ a continuous curve, since in this case (a) is fulfilled ${ }^{2}$ ), (c) $x$ belong to a connected im kleinen subset, $K$, of $B$, such that $x$ is not a limit point of $B-K$.

The accompanying figures are given to show that the two conditions of the above theorem are independent, and that they are only sufficient, not necessary.

Figure 5 is an illustration showing the independence of the conditions $A B C D$ is a square, $E$ the center point of its interior, and $P$ and $F$ the mid-points of $D C$ and $A B$, respectively, $P_{1}, P_{2}, P_{3}, \ldots$ is a sequence of points such that if the distance

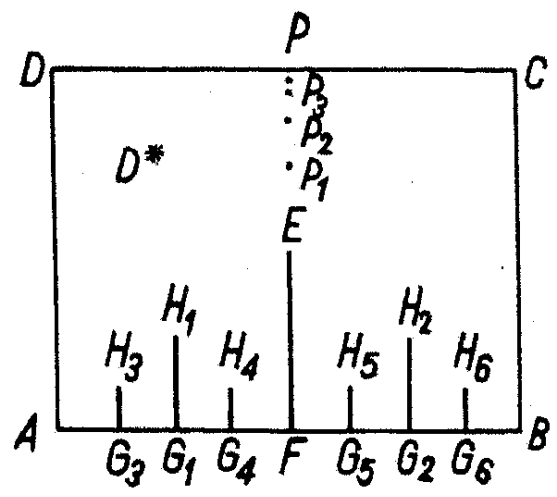
Fig.5 $E P=1 . P_{n}$ lies on the straight line $E P$ and at a distance $1 / n$ from $P . G_{1}$ and $G_{2}$ are the mid-points of $A F$ and $F B$, respectively, and $G_{1} H_{1}$ and $G_{2} H_{2}$ are perpendiculars to $A B$ of length 1/2; $G_{3}, G_{4}, G_{5}$, and $G_{6}$ are mid-points of $A_{1} G_{1}, G_{1} F, F G_{2}, G_{2} B$, respectively, and $H_{3} G_{8}, H_{4} G_{4}, H_{5} G_{5}, H_{6} G_{8}$, are perpendiculars to $A B$ of length $1 / 4$; and so on. Consider the bounded domain $D^{*}$ whose boundary is the set of points consisting of the square $A B C D$, the sequence of points $P_{1}, P_{2} . P_{s}, \ldots$ and the straight line intervals $E F$, $G_{1} H_{1}, G_{2} H_{2}, G_{3} H_{3} ; \ldots$ Every boundary point of $R$ is accessible from any point of $R$; every point of the side $A B$ of $A B C D$ is on

1) 1 will remark here that, if $P$ is a point of $D$, and arcs are taken from $\dot{P}$ to two points $A$ and $B^{\prime}$ of $B$ in such a way that $D$ is divided into two domains $D_{1}$ and $D_{2}, x$ being on the boundary of $D_{1}$, as well as on $B$, that $x$ is accessible from $D_{1}$ provided that the conditions of the theorem are satisfied for $D$, at $x$. That is, the conditions of the theorem are sufficient for accessibility from all sides of the point $x$. I shall not, however, make use of the idea of accessibility from all sides in this paper.

2) Schoenflies showed that all boundary points of an ordinary two-dimensional connected domain are accessible from the domain if its boundary is a continuous curve. Of. A. Schoenflies, Die Entwickelung der Lehre von den Punktmannigjaltigkeiten, Zweiter Teil, Jahresbericht der Deutschen Mathematiker-Vereinigung, vol. 2 (1908), p. 215 
a continuum of condensation of the boundary of $R$, yet condition (1) of Theorem 2 is satisfied for all points of $A B$; condition (1) is not satisfied at $P$, yet $P$ is accessible from any point of $R$ by condition (2).

It can be shown that the above conditions are not necessary by taking as part of the boundary set of isolated points of the domain having $F$ as sequential limit point, somewhat as $P$ is the sequential limit point of the sequence $P_{1}, P_{2}, P_{3}, \ldots$

A point of the boundary may be a limit point of continua of condensation of the boundary and yet be accessible from any point of the domain provided the point does not itself belong to a continuum of condensation of the

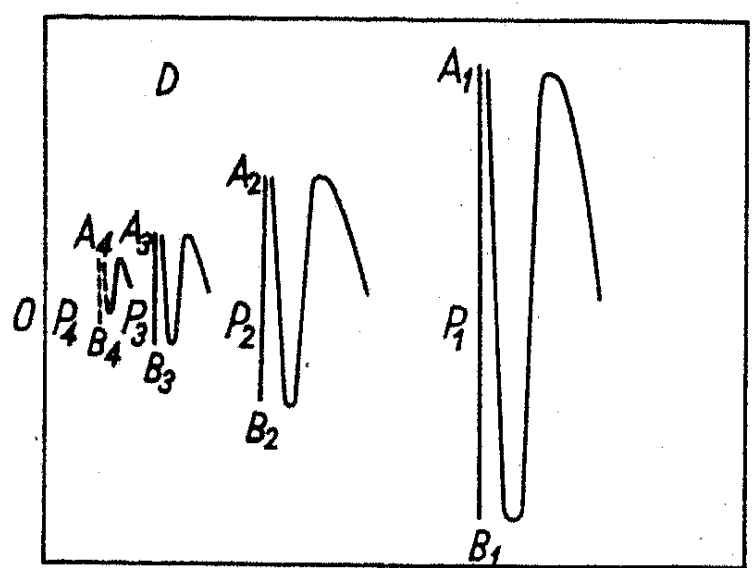

Fig. 6 boundary, as in Figure 6. The points $P_{1}, P_{2}, P_{8}, \ldots$ are situa. ted on the straight line interval $O P_{1}$ in such a way that for every positive integer $n . P_{n}$ is at a distance $1 / n$ from $O$, and $A_{n} B_{n}$ is a straight line interval perpendicular to $O P_{1}$, of length $1 / n$ and mid-point $P_{n}$. The lines $A_{n} B_{n}$ are continua of con. densation of curves of the type $\sin 1 / x$, and $O$ is then a limit point of continua of condensation, but is accessible from any point of $D$.

An extension of space $S$ can be made by considering $S$ to consist of any connected im kleinen, closed and bounded set of points. For this purpose, the following theorem is introduced:

Theorem 3. In order that a closed and bounded point-set should be connected im kleinen, it is necessary and sufficient that it should be the sum of a finite (or vacuous) set of mutually exclusive continuous curves, together with a finite (or vacuous) set of isolated points.

Proof. 1. The condition is necessary. Let $K$ be a closed, bounded and connected im kleinen set of points. By virtue of the property of connectivity im kleinen, every point $P$ of $K$ is the center of two circles $C_{1}$ and $C_{2}$ such that every point of $K$ interior to $C_{2}$ lies with $P$ in some continuum of $K$ that lies entirely interior to $C_{1}$. If $L$ denotes the set of all those points of $K$ interior to $C_{1}$ which lie with $P$ in some continuum of $K$ that lies interior to $C_{1}$, 
together with the limit points of such points, then $L$ is either the isolated point $P$, or a continuous curve which contains every point of $K$ interior to $C_{2}{ }^{1}$ ). Since $K$ is closed and bounded, there exists, by virtue of the Heine-Borel Theorem, a finite number of circles of the type $C_{2}$, namely $C_{2}^{1}, C_{2}^{2}, C_{2}^{3}, \ldots C_{2}^{n}$, such that if $x$ is any point of $K$, there exists a positive integer $i$ such that $x$ lies interior to $C_{2}^{i}$. To each of these circles corresponds a set, $L$, which is either a continuous curve or a single point $P$, and which contains all points of $K$ interior to $C_{2}$. Hence, $K$ consists of a finite (or vacuous) set of continuous curves together with a finite (or vacuous) set of isolated points. Since a finite number of continuous curves which have points in common form one continuous curve, the continuous curves in the theorem can be considered to be mutually exclusive ${ }^{2}$ ).

2. The condition is sufficient. Let $K$ be a closed, bounded set of points which is the sum of a finite 'set of mutually exclusive continuous curves $L_{1}, L_{2}, L_{3}, \ldots, L_{i}$ and a finite set of isolated points $P_{1}, P_{2}, \ldots P_{j}$. If $x$ is a point of the continuous curve $L_{n}$ $(n=1,2, \ldots$ i) there exists a circle $C$ with center at $x$ which contains no points of any other continuous curve of $K$, nor any point of the sequence $P_{1}, P_{2}, \ldots P_{j}$. Since $L_{n}$ is connected im kleinen at $x$, it follows that $K$ is connected im kleinen at $x$. If $x=P_{n}$, $(n=1,2, \ldots j)$, there exists a circle $C$ with center at $x$ containing or enclosing no points of the set $L_{1}+L_{2}+\ldots+L_{n}$. and no point of the sequence $P_{1}, P_{2}, \ldots P_{j}$. The conclusion is obvious.

\section{An analysis of the point-set which constitutes the boun- dary of a complementary domain of a plane continuous curve ${ }^{3}$ ).}

In the present section I shall analyze the boundary of a complementary domain of a plane continuous curve in terms of the elementary untions of point, are and simple closed curve, and prove certain fundamental properties about such sets:

1) Hahn has shown that $L$ is connected in kleinen. $L$ is obviously what Hahn calls $M^{*}(P, r)$. Cf. pg. 2448, loc. cit.

2) Point-sets are satid to be mutually exclusive when they have no points in common.

s) Presented to the American Math. Soc, Dec. 29, 1922. 
If $S$ is a continuous curve in ordinary two-dimensional space $S^{\prime}$, and $P$ a point of $S^{\prime}-S$, that maximal ${ }^{1}$ ) connected subset of $S^{\prime}-S$ determined by $P$ is a complementary domain $D$ of the continuous curve $S$. One of the complementary domains of $S$ is unbounded, and the unbounded complementary domain always exists; $S$ may or may not have bounded complementary domains. The boundary, $\beta$, of $D$, consists of all limit points of $D$ that do not belong to $D$. Evidently $\beta$ is a subset of $S$.

Since $S$ is bounded, there exists a circle $C$, which encloses all points of $S$ but contains no points of $S$. If $D$ is a bounded complementary domain of $S$, then the outer boundary of $D$ is the boundary of the point-set composed of all points $[x]$ such that $x$ can be joined to some point of $C$ by an arc which contains no point of $D+\beta$. R. L. Moore has shown ${ }^{2}$ ) that $B$, the outer boundary of $D$, is a simple closed curve. By a theorem due to Miss Torhorst ${ }^{3}$ ) $\beta$ is itself a continuous curve.

Theorem 4. If $S_{1}$ is the set of all simple closed curves (excluding the outer boundary in case the domain is bounded) contained in the boundary, $\beta$, of a complementary domain of a continuous curve $S$, then (1) $S_{1}$ is countable, (2) if $C_{k}$ and $C_{j}$ are two distinct simple closed curves of the set $S_{1}, C_{k}$ and $C_{j}$ have at most one point in common, and their interiors have no point in common; (3) in case $D$ is bounded and $C$ is any simple closed curve of $S_{1}, C$ lies interiar to $B$, the outer boundary of $D$, or has at most one point $P^{\prime}$ in common with $B$, such that $C-P^{\prime}$ is interior to $B$.

Proof. In case $D$ is bounded, no points of $S_{1}$ can lie exterior to $B$, since all points of $\beta$ must be accessible from $D$ by Theorem 2 .

If $C$ is any closed curve of $S_{1}, C$ cannot have more than one point in common with $B$. For, suppose $C$ has two points, $A$ and $E$, in common with $B$. Then $C$ will contain an arc, $A^{\prime} x E^{\prime}$, which lies, except for $A^{\prime}$ and $E^{\prime}$, wholly interior to $B . A^{\prime} x E^{\prime}$ will divide the

1) If $M$ is a point-set and $P$ a point of $M$, the maximal connected subset of $M$ determined by $P$ is the set of all points of $M$ that lie, with $P$, in a connected sabset of $M$.

2) R. L. Moore, Concerning continuous curves in the plane, Math. Zeitschr. Band 15, (1922) pp. 254-260.

I) Ueber den Rand der einfach zusammen hängeng" ebenen Gebiete. Math. Zeitschr. 9 (1921), S. 64 (73). 
interior of $B$ into two domains, $R_{1}$ and $R_{2}$, which are mutually exclusive ${ }^{1}$ ), and such that if $I$ is the interior of $B$,

$$
I=R_{1}+R_{2}+A^{\prime} x E^{\prime}-A^{\prime}-F^{\prime} .
$$

$D$ must lie wholly in either $R_{1}$ or $R_{2}$. Clearly points on one of the ares into which $A^{\prime}$ and $E^{\prime}$ divide $B$ are not accessible from $D$, a contradiction of Theorem 2. Hence $C$ can have at most one point, $P$, in common with $B$, and the set $C-P$ must lie interior to $B$.

In any ease, $\boldsymbol{D}$ being bounded or unbounded, if $C_{\boldsymbol{k}}$ and $C_{j}$ are two distinct simple closed curves of the set $S_{1}$, the interiors of $C$ and $C_{s}$ can have no point in common; for if their interiors have a point in common, points of $C_{k}$ lie interior to $C_{j}$, or vice versa, and a contradiction of Theorem 2 results.

Furthermore, $C_{k}$ and $C_{j}$ can not have more than one point in common. For suppose they have two points, $A$ and $E$, in common. $C_{j}$ must contain at least one point, $x$ which is exterior to $C_{k}$, since $C_{k}$ and $C_{j}$ are not identical and their interiors have no point in common, and there will exist two points, $A^{\prime}$ and $E^{\prime}$ : common to $C_{k}$ and $C_{j}$, such that the arc $A^{\prime} x E^{\prime}$ is exterior to $C_{k}$. From Theorem 27, of R. L. Moore's Foundations of Plane Analysis Situs, it follows that one of the ares into which $A^{\prime}$ and $E^{\prime}$ divide $C_{k}$ is interior (except for end-points) to the simple closed curve formed by the other arc of $C_{k}$ and $A^{\prime} x E^{\prime}$, a contradiction of Theorem 2 again.

$S_{1}$, then, is a totality of simple closed curves whose interiors have no point in common, and such that any two have not more than one point in common. The closed curves of this set must therefore form the boundaries of a set of mutually exclusive domains. As every set of mutually exclusive domains is countable, $S_{1}$ must be a countable set $C_{1}, C_{2}, C_{3} \ldots$

Theorem 5. The boundary, $\beta$, of $\dot{a}$ complementary domain, $D$, of a continuous curve, $S$. cannot contain an uncountable infinity of simple continuous arcs no two of which have a point in common.

Proof. Suppose that $\beta$ does contain an uncountable set, $T$, of simple continuous arcs, such that no two ares of $T$ have a point in common. Then there exists some positive number $\varepsilon$. such that there

1) Cf. R. L. Moore, Foundations ${ }^{\prime} f$ plane Analysis Situs, loc. cit. p. 141. 
are incountably many arcs of $T$ of diameter ${ }^{1}$ ) greater than $\varepsilon$. Call the totality of these $T^{\prime}$.

In each arc of $T^{\prime}$ there are two points $x$ and $y$ such that

$$
\delta(x, y)>\varepsilon \text {. }
$$

Assign $x$ to a set $[A]$ and $y$ to a set $[B]$. The set $[A]$ will have at least one limit point, $A$, since it is bounded. Then from $[A]$ can be selected a sequence of points $x_{1}, x_{2}, x_{3}, \ldots$ which bas $A$ as a sequeutial limit point. Let $y_{1}, y_{2}, y_{3}, \ldots$ be points of $[B]$ such that for every positive integer $n ; x_{n}$ and $y_{n}$ are points of the same arc of $T^{\prime}$.

The set $y_{1}, y_{2}, y_{3}, \ldots$ must have at least one limit point, $B^{*}$, and contains a subsequence $B_{1}, B_{2}, B_{3}, \ldots$ which has $B^{* *}$ as a sequential limit point. Let $A_{1}, A_{2} . A_{3}, \ldots$ be a subsequence of the sequence $x_{1}, x_{2}, x_{3}, \ldots$ such that for every positive integer $n, A_{n}$ and $B_{n}$ belong to the same arc of the set $T^{\prime}$. Then $A$ and $B^{*}$ are sequential limit points of the sequeuces $A_{1}, A_{2}, A_{3}, \ldots$ and $B_{1}, B_{2}, B_{3}, \ldots$, respectively, since if a sequence of points has a sequential limit point, every subsequence of it has the same sequential limit point. $A$ and $B^{*}$ are distinct points, since, as a result of (1),

$$
\delta\left(A, B^{*}\right) \geqq \varepsilon
$$

Let

$$
\delta\left(A, B^{\ddot{*}}\right)=8 \eta \text {. }
$$

Hahn has shown ${ }^{2}$ ) that a connected im kleinen continuum is uniformly connected im kleinen. That is, for every positive number $\varepsilon$ there exists a positive number $\delta_{\varepsilon}$, such that if $P^{\prime}$ and $P^{\prime \prime}$ are two points of the continuum such that

$$
\delta\left(P^{\prime}, P^{\prime \prime}\right)<\delta_{\varepsilon}
$$

then $P^{\prime}$ and $P^{\prime \prime}$ are the extremities of an arc, $L$, such that if $x^{\prime}$ is any point of $L$.

and

$$
\delta\left(x^{\prime}, P^{\prime \prime}\right)<\varepsilon
$$

$$
\delta\left(x^{\prime}, P^{\prime \prime}\right)<\varepsilon
$$

1) The diameter of a point-set $M$ is the upper limit of $\delta_{y}(x, y)$. where $x$ and $y$ are any two points of $M$ and $\delta$ meims distance; i. $\theta ., \delta(x . y)$ stands for the distance from $x$ to $y$.

\%) Loc. cit. 
Since $\beta$ is a continuous curve, and therefore a connected in kleinen continuum, it is uniformly connected im kleinen.

Therefore, corresponding to the number $\eta$ there exists a number $\delta_{\eta}$ satisfying the conditions for uniform connectivity im kleinen.

There exists a positive number $N$ such that if $n>N, A_{n}$ is interior to a circle of diameter $\delta_{\eta \eta}$ with $A$ as center, and $B_{n}$ is interior to a circle of diameter $\delta_{\eta}$ with $B^{*}$ as center. Hence, for any such value of $n$,

and

$$
\delta\left(A_{n}, A_{n+1}\right)<\delta_{\eta}
$$

$$
\delta\left(B_{n}, B_{n+1}\right)<\delta_{\eta} .
$$

Then $A_{n}$ and $A_{n+1}$ are the extremities of an $\operatorname{arc}$ of $\beta$ such that if $P$ is a point of this arc,

and

$$
\delta\left(P, A_{n}\right)<\eta .
$$

$$
\delta\left(P, A_{n+1}\right)<\eta \text {; }
$$

and, since $\delta_{\eta} \leqq \eta$,

$$
\delta(P, A)<2 \eta
$$

Similarly, for $n>N, B_{n}$ and $B_{n+1}$ are the extremities of an are of $\beta$ - satisfying similar conditions. These tro arcs can have no point in common, and the arcs $A_{n} B_{n}$ and $A_{n+1} B_{n+1}$ bave no point in common by hypothesis. From the point set composed of these four arcs can be selected a simple closed curve which consists of intervals of the four arcs. This simple closed curve bounds a do. main which is bounded. The totality of such domains form a sequence $D_{1}, D_{2}, D_{3}, \ldots$, each of whose boundaries belongs to $S_{1}$ (except for one which may be the outer boundary, $B$, and which, for convenience, may be considered as omitted from the sequence). By Theorem 4 (2), these domains are mutually exclusive; furthermore, they constitute an infinite set of domains complementary to $\beta$, each of which is of diameter greater than $4 \eta$. But this is a contradiction of a theorem due to Schoenflies ${ }^{1}$ ) to the effect that if $\varepsilon$ is any given positive number, there do not exist infinitely many domains complementary to a continuous curve, each of which is of diameter greater than $\varepsilon$

1) Loc. cit., p. 221, IX. 
Hence $\beta$ cannot contain uncountably many arcs no two of which have a point in common.

Theorem 6. The boundary, $\beta$, of a complementary domain of a continuous curve, cannot contain an infinite set of simple continuous arcs of diameter greater than any positive number $\varepsilon$, such that no two of these arcs have an interior point of both in common.

Proof. If such a set exists, select from it a sequence $t_{1}, t_{2}, t_{3}, \ldots$ For every positive integer $n, t_{n}$, being of diameter greater than $\varepsilon$, must contain an arc $t_{n}^{\prime}$ which does not contain the end points of $t_{n}$ and is itself of diameter greater than $\varepsilon$. Then the set $t_{1}^{\prime}, t_{2}^{\prime}, t_{3}^{\prime}, \ldots$ is an infinite sequence of ares no two of which have a point in common, and each of which is of diameter greater than $\varepsilon$ An argument similar to that used in the proof of Theorem 5 may be used to complete the proof of this Theorem.

Definition: If $M$ is a continuous curye, I shall define an end-point of $M$ to be any point $P$ of $M$, such that if $a$ is an arc of $M$ whose end-points are $P$ and any other point $P^{\prime}$ of $M$, the set. $M-$ - $(a-P)$ contains no connected subset consisting of more than one point which contains $P$. If a point $P^{\prime}$ can be found such that this condition is not satisfied, then $P$ is not au end-point of $M$.

Definition: If $M$ is a continuous curve, and $P$ a point of $M$, then if $M-P$ is not connected, $P$ is called a cut-point ${ }^{1}$ ) of $M$. If $M-P$ is connected, I shall call $P$ a non-cut-point of $M$.

Theorem 7. In order that a point of a continuous curve that contains no simple closed curves should be an end-point, it is necessary and sufficient that it be a non-cut-point.

-Proof. (a) The condition is necessary. For, let $P$ be an end-point of a continuous curve $M$ which contains no simple closed curve, and let $P^{\prime}$ be any other point of $M . P$ and $P^{\prime}$ are the extremitie of an arc $a$ of $M$. Since $P$ is an end point, $M-(a-P)$ contains no connected subset consisting of more than one point that contains $P$. Suppose that $P$ is a cut-point of $M$ Then

$$
M \cdot P=M_{1}+M_{2}
$$

1) This definition was given by R. L. Moore in Concerning the cul-points of continuous curves and of other closed and connected point-sets. Proc. Nat. Acad. Sci vol. IX. (1923), pp, $101-106$ 
where $M_{1}$ and $M_{2}$ are two non-vacuous mutually separated ${ }^{1}$ ) sets. Now $P$, being an end-point of $a$, does not disconnect $a$. And $a-P$, being connected, must lie wholly in $\boldsymbol{M}_{1}$ or $M_{\mathbf{2}}$. Suppose it lies in $M_{1}$. Since $M_{1}$ has no limit points in $M_{2}, M_{2}$ contains a domain $d$ with respect to $M$, whose boundary with respect to $M$ is the point $P$. If $x$ is a point of $M_{2}, x$ and $P$ are the extremities of an arc $a^{\prime}$ which lies wholly in $d$ and hence in $M_{2}$, except for the point $P$, by Theorem 1 . But since $a-P$ lies wholly in $M_{1}$,

$$
\left.M-(a-P) \supset M_{2}+P \supset a^{\prime 2}\right)
$$

That is, $M-(a-P)$ contains a connected subset consisting of more than one point which contains $P$. Hence under the supposition that $P$ is a cut-point of $M$ a contradiction results. Therefore $P$ must be a non-cut-point of $M$.

(b) The condition is sufficient. Let $P$ be a non-cut-point of a continuous curve $M$ that contains no simple closed curve. Suppose $P$ is not an end-point of $M$. Then there exists a point. $P^{\prime}$ of $M$, such that if $a$ be an are of $M$ whose extremities are $P$ and $P^{\prime}$, $M-(a-P)$ contains a connected subset $M_{\mathbf{2}}$, consisting of more than one point, which contains $P$.

Since $P$ is a non-cut-point, $M-P$ is connected. Divide $M-P$ into two sets, $a-P$ and $M_{2}^{\prime}$. Then

$$
M_{2}^{\prime} \supset M_{2}-P \text {. }
$$

Let $d$ be that maximal connected domain with respect to $M$ such that

$$
M_{2}^{\prime} \supset d
$$

and

$$
d \supset M_{3}-P \text {. }
$$

Then $d$ must have some limit point $x$ distinct from $P$ in $a$.

Let $y$ be a point of $d$. Thère exists an are $a^{\prime}$ whose extremities are $x$ and $y$ and which lies wholly in $d$ except for $x$ (see Theorem 1); also an arc $a^{\prime \prime}$ whose extremities are $y$ and $P$, and which lies wholly in $d$ except for $P$. Then $a, a^{\prime}$ and $a^{\prime \prime}$ contain a simple closed curre. But $M$ contains no simple closed curve by hypothesis.

1) Two point-sets are said to be malually separated when they are mutually exclusive and neither contuins a limit point of the other.

2) The symbol $\supset$ should bo read "contains or is identical with". 
Hence the supposition that $P$ is not an end-point of $M$ leads to a contradiction, and $P$ must therefore be an end-point of $M$.

Theorem 8. If $M$ is a continuous curve that contains no simple closed curve, then $M$ cannot contain. for any given positive number $\varepsilon$, an infinite number of arcs of diameter greater than $\varepsilon$, and such that no two of these arcs have an interior point of both in common.

Proof: The proof of this theorem is nearly identical with the proofs of Theorems 5 and 6 , except that a contradiction is obtained as soon as it is demonstrated that $M$ contains a single simple closed curve. Or, it may be considered a special case of Theorem 6, since such a continuous curve is the boundary of only one domain, namely, its unbounded complementary domain.

Theorem 9. If $M$ is a continuous curve, and $N$ is a closed proper ${ }^{1)}$ subset of $M$, then $M-N$ is a countable set of domains with respect to $M$ whose boundary points with respect to $M$ are contained in $N$.

Proof: Let $P$ be a point of $M-N$. Then that maximal connected subset, $d$, of $M-N$ determined by $P$ is a domain with respect to $M$. To show this, let

$$
R=M-(N+d)
$$

Then $R$ and $d$ are mutually separated. For, $R$ can contain no limit point, $x$, of $d$, since $d+x$ would be connected and $x$ therefore a point belonging to $d$, contrary to (1). On the other hand, if $d$ contains a limit point, $y$, of $R$, there exist two circles, $k_{1}$ and $k_{2}$, with centers at $y$, neither of which encloses a point of $N$, and such that every point of $M$ interior to $k_{2}$ lies with $x$ in a connected subset of $M$ that lies wholly interior to $k_{1}$. and furthermore such that at least one point, $x$, of $R$. lies within $k_{2}$. Then $x$ and $y$ lie in a connected subset $L$ of $M$ that lies wholly interior to $k_{1}$. But no points of $N$ lie interior to $k_{1}$, and therefore

$$
M-N \supset L \text {. }
$$

As $d$ is a maximal connected subset of $M-N, y$ must belong to $d$. This is impossible, since $R$ and $d$ are mutually exclusive according to (1). Hence no points of $R$ can lie interior to $k_{2}$ and $y$

1) If $N$ is a subset of a pointwset $M$, then $N$ is a proper subset of $M$ if $M-N$ is not vacuous. 
cannot be a limit point of $R$. As $R$ contains no limit points of $d$, and $d$ contains no limit points of $R$, and as, moreover, the two sets are mutually exclusive by (1), $R$ and $d$ are mutually separated.

Hence, if $P^{\prime}$ is any point of $d$, there exists a circle $C_{1}$ which encloses $P^{\prime}$, but no points of $R$ or $N$. It follows at once that $d$ is a domain with respect to $M$. The boundary of $d$ with respect to $M$ must be a subset of $N$, since $d$ can have no limit points in $R$.

Since every point $P$ of $M-N$ determines a maximal connected subset of $M-N$, i. e., a domain with respect to $M, M-N$ is a totality of domains, $[d]$, with respect to $M$, whose boundaries with respect to $M$ belong to $N$.

To show that these domains form a countable set, it is only necessary to make use of the fact that every uncountable point-set contains at least one of its limit points. For, if $Q$ be a point set which consists of points of $[d]$ such that one and only one point of each domain of $[d]$ belongs to $Q, Q$ contains at least one limit point of itself. Call such a point $A$. Let $d$ be that domain of $[d]$ of which $A$ is a point. If $R$ be defined as in (1), then

But

$$
R \supset[d]-d \text {. }
$$

$$
[d]-d \supset Q-A \text {. }
$$

From (2) and (3) it follows that $A$ is a limit point of $R$, which is impossible, as shown above. Hence the set of domains $[d]$ is countable.

Theorem 10. If $M$ is a continuous curve that contains no simple closed curve, and $N$ a closed proper subset of $M$, then for any positive number $\varepsilon, M-N$ contains at most a finite number of maximal domains with respect to $M$ of diameter greater than $\varepsilon$.

This theorem is a direct consequence of Theorems 8 and 9 , and the fact that every two points of a domain with respect to a continuous curve are the extremities of an arc of that domain.

Theorem 11. Every closed and connected subset of the boundary of a complementary domain of a continuous curve is itself a continuous curve.

Proof: Let $\beta$ be the boundary of a complementary domain of a continuous curve $S$, and $N$ a closed and connected subset of $\beta$. To show that $N$ is a continuous curve, it is necessary to prove it connected im kleinen. 
Let $P$ be a point of $N$ at which it is not connected im kleinen. Then there will exist ${ }^{1}$ ) two concentric circles $k_{1}$ and $k_{2}$, and a countable infinity of closed and connected point-sets, $M, M_{1}, M_{2}, M_{3}, \ldots$ such that (1) each of these point-sets is a subset of $N$ and contains at least one point on $k_{1}$ and at least one point on $k_{2}$, but contains no point exterior to $k_{1}$ or interior to $k_{2}$, (2) no two of these point-sets have a point in common, and indeed, no one of them is a proper subset of any other connected subset of $N$ which contains no point without $k_{1}$ or within $k_{2},(3)$ the set $M$ is the sequential limiting set ${ }^{2}$ ) of the sequence of sets $M_{1}, M_{2}, M_{8}, \ldots$

Let $\varrho$ be the lower limit of the distance $x y$, where $x$ is any point of $M_{2}$ and $y$ is a point of $M_{1}+M_{8}$. Since $\beta$ is a continuous curve and therefore uniformly connected im kleinen, there exists a number $\eta$ such that if $a$ and $b$ are any two points of $\beta$, such that

$$
\delta(a, b)<\eta
$$

then $a$ and $b$ arc the extremities of an $\operatorname{arc} a b$ of $\beta$, such that every. point $c$ of the arc $a b$ satisfies the relations

$$
\begin{aligned}
& \delta(a, c)<\varrho \\
& \delta(b, c)<\varrho
\end{aligned}
$$

Since $M_{2}$ is a continuum, there exists in $M_{2}$ a chain of points $x_{1}, x_{2}, \ldots x_{k}$, such that

$$
\delta\left(x_{i}, x_{i+1}\right)<\eta, \quad(i=1,2, \ldots k-1)
$$

$x_{1}$ is a point of $k_{1}$, and $x_{k}$ a point of $k_{2}$. Then there exists an arc $x_{i} x_{i+1}$ of $\beta$, such that if $x^{\prime}$ is any point of this arc,

$$
\begin{gathered}
\delta\left(x_{i}, x^{\prime}\right)<\rho \\
\delta\left(x_{i+1}, x^{\prime}\right)<\rho .
\end{gathered}
$$

1) Cf. $\mathbf{R}$ L. Moore, A characterization of Jordan regions by properties having no reference to their boundaries, Proc. Nat. Acad. Sci., IV (1918), pp. $364-370$.

2) A point-set $t$ is said to be the sequential limiting set of a sequence of point-sets $t_{1}, t_{2}, t_{3}, \ldots$ provided that (a) each point of $t$ is the sequential limit point of an infinite-sequence of points, $P_{1}, P_{2}, P_{3} \ldots$ such that, for every $n, P_{n}$ belongs to $t_{n}$, and (b) if $P_{1}, P_{2}, P_{3}, \ldots$ is a sequence of points such that, for every $n$, $P_{n}$ belongs to $t_{n}$ then $t$ contings the sequential limit point af every subsequence of $P_{1}, P_{2}, P_{8}, \ldots$ which has a sequential limit point. 
The set $x=\Sigma x_{i} x_{i+1}$ is a continuous curve, since any connected set consisting of a finite number of ares is a continuous curve, and therefore contains an arc whose end-points are $x_{1}$ and $x_{k}$. Let $x_{1}^{\prime}$ be the last point of this are on $k_{1}$ in the order from $x_{1}$ to $x_{k}$, and $x_{k}^{\prime}$, the first point of this are on $k_{z}$ in the order from $x_{1}^{\prime}$ to $x_{k}$. Then $x_{1}^{\prime} x_{k}^{\prime}=t_{2}$ is an arc of $\beta$ which lies. except for ils end-points, wholly interior to $k_{1}$ and wholly exterior to $k_{2}$.

Now let $\varrho^{\prime}$ be the lower limit of the distance $x y$, where $x$ is any point of $M_{3}$, and $y$ any point of $M_{4}+t_{2}$. There exists a number $\eta^{\prime}$ such that if $a$ and $b$ are any two points of $\beta$ such that

$$
\delta(a, b)<\eta^{\prime}
$$

then $a$ and $b$ are the extremities of an arc $a b$ of $\beta$, such that every point $c$ of the are $a b$ satisfies the relations

$$
\begin{aligned}
& \delta(a, c)<\varrho^{\prime} \\
& \delta(b, c)<\varrho^{\prime},
\end{aligned}
$$

As $M_{3}$ is a continuum, there exists in $M_{3}$ a chain of points $\bar{x}_{1}$, $\bar{x}_{2}, \ldots \bar{x}_{n}$, such that

$$
\left.\delta \overline{\left(x_{i}\right.}, \bar{x}_{i+1}\right)<\eta^{\prime}, \quad(i=1,2, \ldots n-1)
$$

$\overline{x_{1}}$ is a point of $k_{1}$, and $\bar{x}_{n}$ a point of $k_{2}$. By a discussion similar to that used above in showing the existence of the arc $t_{2}$, it can be proved that there exists an arc $t_{9}$, having properties similar to those of $t_{2}$.

Continuing in this manner indefinitely, it can be shown that there exist an infinite sequence of arcs, $t_{2}, t_{3}, t_{4}, \ldots$ such that for every positive integer $n>1$,

$$
\beta \supset t_{n},
$$

the diameter of $t_{n}$ is $>r / 2$, where $r$ is the numerical difference of the radii of $k_{1}$ and $k_{2}$ and such that $t_{n}$ has no point in common with any other are of the sequence $t_{2}, t_{3}, t_{4}, \ldots$ Clearly this is a contradiction of Theorem 6 . Hence $N$ must be connected in kleinen at $P$, and as $P$ is any point of $N . N$ must be connected im kleinen.

It might be noted in passing that Theorem 11 is a generalization of a result oblained by Mazurkiewicz ${ }^{1}$ ) to the effect that every

1) S. Mazurkiewicz, Un théorème sur les lignes de Jordan, Fund. Math., II, (1921) pp. $123-125$. 
closed and connected subset of a continuous curve that contains no simple closed curve is itself a continuous curve.

Theorem 12. If $M$ is a continuous curve that contäins no simple closed curve, $N$ a closed and connected proper subset of $M$, and $d$ a maximal connected domain which is a subset of $M-N$, then $d$ has only one boundary point with respect to $M$, and that point belongs to $N$.

Proof: The boundary points of $d$ all lie in $N$ as a consequence of Theorem 9. Suppose there exist two boundary points of $d$ with respect to $M$. By Theorem $11 N$ is itself a continuous curve and therefore contains an are from $P_{1}$ to $P_{2}$. But by application of Theorem 1 there exists another arc from $P_{1}$ to $P_{2}$ which lies, except for $P_{1}$ and $P_{2}$, wholly in $d$. As $d$ and $N$ have no points in common, these two arcs have in common only the points $P_{1}$ and $P_{2}$, and their sum is therefore a simple closed curve. But this is a contradiction of the hypothesis that $M$ contains no simple closed curve. Hence $d$ has only one boundary point with respect to $M$.

For convenience, the major results of Theorem 9,10 and 12 are embodied in one Theorem as follows:

Theorem 13. If $M$ is a continuous curve that contains no closed curve, and $N$ is a closed and connected proper subset of $N$, then $M-N$ consists of a countable set of domains with respect to $M$, viz., $d_{1} . d_{3}, d_{3}, \ldots$ such that (1) for every positive integer $n, d_{n}$ has one and only one boundary point with respect to $M$, and this point is a point of $N$, (2) no two of these domains have a point in common, and (3) if $\varepsilon$ is any positive number, at most a finite number of these domains are of diameter greater than $\varepsilon$

Theorem 14. If $x$ and $y$ are two distinct points of a continuous curve $M$ that contains no simple closed curve, then no point of the arc $x y$ of $M$, excepting the points $x$ and $y$, is an end-point of $M$.

Proof: As shown by Mazurkiewicz ${ }^{1}$ ), there exists in $M$ only one arc from $x$ to $y$. Let $P$ be a point of $x y$, distinct from $x$ and $y$. Then

$$
x y-P=M_{1}+M_{2},
$$

where $M_{1}$ and $M_{2}$ are mutually separated sets. As a consequence of Theorem $13 M-x y$ consists of a set of domains with respect to $M$ each of which has one and only one boundary point with

1) See Un théorème sur les lignes de Jordan. Loc, cit. 
respect to $M$, that point being a point of $x y$. Let $\left[d^{\prime}\right]$ be the collection of all those domains of $M-N$ whose boundary points with respect to $M$ lie in $M_{1}$, and . $\left[d^{\prime \prime}\right]$ the collection of all those domains of $M-N$ whose boundary points lie in $M_{2}$.

If $P$ is the boundary with respect to $M$ of a domain $d_{i}$, then $d_{i}$ and $M-\left(d_{i}+P\right)$ are two mutually separated sets (see Theorem 13), whence by Theorem $7 P$ is not an end-point.

If $P$ is not the boundary with respect to $M$ of any domain, then the sets $\left[d^{\prime}\right]+M_{1}$ and $\left[d^{\prime \prime}\right]+M_{2}$ are mutually separated, and $P$ is not an end-point of $M$.

In any case then, $P$ cannot be as end-point.

Theorem 15. A continuous curve $M$ that contains no simple closed curve consists of (1) a sequence of arcs $c_{1}, c_{2}, c_{3}, \ldots$ no two of which have in common an interior point of both. and suich that (i) if $n$ is any positive integer, $c_{1}+c_{2}+c_{3}+\ldots+c_{n}$ is a continuous curve $M_{n}$ and: a proper subset of $M$, (ii) for $\varepsilon>0$, there exists a number $\varrho$ such that it $n>\varrho$,

$$
\delta\left(c_{n}\right)<\varepsilon,
$$

and the diameter of any one of the countable set of maximal domains with respect to $M$ lying in $M-M_{n}$ is less than $\varepsilon$; and (2) $a$ totally disconnected set of end-(or non cut-) points, $P_{\omega}$, each of which is a limit point of the sequence $M_{1}, M_{2}, M_{3}, \ldots$ and which contains all the end- (or non-cut-) points of $M$.

Proof: The continuous curve $M$ is of diameter greater than some pusitive number. say, 1. Then there exist two points, $x$ and $y$ belonging to $M$, that are not end-points of $M$, and whose distance apurt is greater than 11 ). Let $c_{1}$ be that are of $M$ which has $x$ and $y$ as end-points. As a result of Theorem 13, $M-c_{1}$ is a set of domains with respect to $M$, whose boundary points with respect to $M$ lie on $c_{1}$, and such that only a finite number of these domains, $d_{2}, d_{3}, d_{4}, \ldots d_{k}$. are of diameter greater than 1 . Let $P_{i}$ be the boundary point of $d_{i}$ with respect to $M(i=2,3 \ldots k)$. Since the

1) That two such points cin be found is a direct consequence of a theorem proved by R. L. Moore, viz., in order that a closed, connected and bounded point-set $M$ shoull be a continuous curve which contains no simple closed curre, it is necessary and sufticient that every closed and connected subset of $\boldsymbol{M}$ should contain uncountahly many cut-points; (See R. L. Moore, Concerning the cul-points of continuous curves and of other closed and connected point-sets, loc. cit. Th. E). and of 'Theorem 7 . 
diameter of $d_{i}$ is greater than 1 , there exist in $d_{i}$ two points $x_{i}$ and $y_{\imath}$ such that

$$
\delta\left(x_{i}, y_{i}\right)>1
$$

It is clear, then, that either

$$
\delta\left(P_{i}, x_{i}\right)>\frac{1}{2}
$$

or

$$
\delta\left(P_{i}, y_{i}\right)>\frac{1}{2}
$$

Suppose that

$$
\delta\left(P_{i}, x_{i}\right)>\frac{1}{2}
$$

Then the set composed of $d_{i}$ and $P_{i}$ contains an arc from $x_{i}$ to $P_{i}$ whose diameter is greater than $\frac{1}{2}$.

Now the set

where

$$
M_{1}^{*}=c_{1}+c_{2}+\ldots+c_{k}
$$

$$
c_{i}=\operatorname{arc} x_{i} P_{i} \quad(i=2,3, \ldots k)
$$

is a continuum. Then $M-M_{1}^{*}$ consists of a set of domains ${ }^{1}$ ) only a finite number of which can be of diameter greater than 1; let these be $d_{k+1}, d_{k+2}, \ldots d_{k+j}$. Since .

$$
\left.\delta\left(d_{k+i}\right)>1, \quad(i=1,2,3, \ldots, j)^{2}\right)
$$

there exist in $d_{k+i}$ two points $x_{i}$ and $y_{i}$, which are not end points, such that

$$
\delta\left(x_{i}, y_{i}\right)>1 \text {. }
$$

Then, if $P_{i}$ be the boundary point of $d_{k+i}$ with respect to $M$,

$$
\delta\left(P_{i}, x_{i}\right)>\frac{1}{2}
$$

or

$$
\delta\left(P_{i}, y_{i}\right)>\frac{1}{2}
$$

1) $M-M_{1}^{*}$ is not vacuous, since $M_{1}^{*}$ contains no end-points by Theorem 14 (the points $x_{i}$ and $P_{i}$ being also non-cut points) and it has been shown by $\mathrm{R}$. L. Moore that every bounded continum contains at least two non-cut points. Cf. R. L. Moore, Concerning stmple continuous curves. Transactions of the American Mathematical Society, vol. 21 (1920), pp. 340-341, Theorem 2; Concerning the cut-points of continuous curves and of other closed and connected point-sets, loc. cit. Also seo 8. Mazurkiewicz, Un théorème sur les lignes de Jordan loc. cit. pp. 119-130.

2) Where the notation for a point-set is placed in the parenthesis, the $\delta$ should be rearl "the diameter of ${ }^{*}$. 
Suppose that

$$
\delta\left(P_{i}, x_{i}\right)>\frac{1}{2} .
$$

Then by virtue of Theorem $1, d_{k+i}+P_{i}$ contains an arc $c_{k+i}$ whose extremities are $x_{i}$ and $P_{i}$. Thus a continuum

is obtained.

$$
M_{2}^{*}=M_{1}^{*}+c_{k+1}+\ldots+c_{k+j}
$$

Continuing this process, there is obtained, eventually, a continuum

$$
M_{m}^{*}=c_{1}+c_{2}+\ldots+c_{h}
$$

such that $M-M_{m}^{*}$ consists of a set of domains only a finite number of which are of diameter $>\frac{1}{2}$, and none of which is of diameter $>1$. For if domains of diameter $>1$ could be obtained indefinitely, plainly an infinite set of arcs of diameter $>\frac{1}{2}$ would also be obtained, a situation contradictory to Theorem 8 . $M-M_{m}^{*}$ being non-vacuous, let its domains of diameter $>\frac{1}{2}$, if any exist, be denoted by $d_{n+1}, d_{h+2}, \ldots d_{n+q}$. Let the boundary point of $d_{k+i}$ $(i=1,2, \therefore q)$ be $P_{i}$. Since

$$
\delta\left(d_{n+i}\right)>\frac{1}{2}
$$

it can be shown that there exists in $d_{h+i}$ a point $x_{i}$ such that

$$
\delta\left(P_{i}, x_{i}\right)>\frac{1}{4},
$$

and hence $d_{n+i}+P_{i}$ contains an arc $c_{n+i}$ from $P_{i}$ to $x_{i}$.

If the process indicated above be continued indefinitely, there is obtained a con zcted set of ares $c_{1}, c_{2}, \ldots$, such that (1) none of these arcs contains any end-point of $M$ by virtue of Theorem 14, and no two of them have in -common a point which is an interior point of both, (2) if $\varepsilon$ is any positive number, there exists a number $\eta$ such that if $n$ is any positive integer $>\eta$,

$$
\delta\left(c_{n}\right)<\varepsilon,
$$

and the diameter of every one of the countable set of maximal domains with respect to $M$ lying in $M-M_{n}$, where

$$
M_{n}=c_{1}+c_{2}+\ldots+c_{n}
$$

(a continuous curve since it consists of a finite and connected set of ares) is less than $\varepsilon$.

If the set of points consisting of the totality of arcs in the sequence $c_{1}, c_{2}, c_{3}, \ldots$ be denoted by $M^{*}$, then $M^{*}$ is non-vacuons. 
For $M^{*}$ contains no end-points of $M$ by (1) above, and $M$ must contain at least two end-points, as already indicated.

Every point of $M-M^{*}$ is a limit point of $M^{*}$. For, suppose $P$ is a point of $M-M^{*}$ that is not a limit point of $M^{*}$. Then there exists a circle $k$ with center $P$ that contains no point of $M^{*}$. But $P$ must belong to some proper connected subset $s$ of $M-M^{*}$ that lies interior to $k$, by virtue of the properties of connectivity and connectivity im kleinen of $M$. Let $\varrho$ denote a positive number such that

$$
\delta(s)>\varrho .
$$

Now there exists a number $\eta$, such that if $n$ is any positive number $>\eta, M-M_{n}$ consists of a set of maximal domains with respect to $M$ no one of which is of diameter greater than $\varrho$. However, $s$ must belong to some domaiu of $M-M_{n}$, since

$$
M-M_{n} \supset M-M^{*} \supset s,
$$

and this domain cannot be of diameter greater than $\varrho$. Therefore the supposition that $P$ is not a limit point of $M^{*}$ leads to a contradiction; hence all points of $M-M^{*}$ must be limit proints of $M^{*}$.

The set $M-M^{*}$ is a totally disconnected set, since if it contains a connected subset $s$ a contradiction will result as in the preceding paragraph.

Since $M^{*}$ contains no end-points of $M$, and since $M$ must have at least two end-points; all end-points of $M$ must lie in $M-M^{*}$. It remains to show that every point of $M-M^{*}$ is an end-point of $M$.

Suppose there exists in $M-M^{*}$ a point $P$ that is not an end-point of $M$, then $P$ is a cut-point of $M$ by virtue of Theorem 7 . Hence

$$
M-P=H_{1}+H_{2},
$$

where $H_{1}$ and $H_{2}$ are mutually separated sets. $M^{*}$ is a connected set that does not contain $P$, and therefore $P$ cannot disconnect $M^{*}$. Hence $M^{*}$ must lie wholly in $H_{1}$ or $H_{2}$, say in $H_{1}$. As $H_{2}$ cannot contain any limit points of $H_{1}$. and as all points of $M-M^{*}$ are limit points of $M^{*}$, the set $M-\left(M^{*}+P\right)$ must be a subset of $H_{1}$.

But

Hence, if

$$
M-P=M^{*}+M-\left(M^{*}+P\right) \text {. }
$$

$$
H_{1} \supset M^{*}+M-\left(M^{*}+P\right)
$$


$\mathrm{H}_{2}$ must be a vacuous set. That is, $M-P$ does not allow of division into mutually separated sets, and is therefore connected. $P$ is therefore a non-cut point of $M$, and by Theorem 7 an end-point of $M$. This completes the proof of the Theorem, the set $M-M^{*}$ being the set $P_{\omega}$.

The following theorem is a generalization of Theorem 13:

Theorem 16. If $M$ is the boundary of a complementary domain of a continuous curve, and $N$ a closed and connected proper subset of $M$, then $M-N$ consists of a countable set of domains with respect to $M$, viz, $d_{1}, d_{2}, d_{3}, \ldots$ satisfying the conditions of Theorem 13, except that (1) should be made to read, ${ }_{n}$ for every positive integer $n, d_{n}$ has at most two boundary points with respect to $M$, ind these points belong to $N^{u}$.

The proof is very similar to the proof of Theorem 13 .

Theorem 17. The boundary of a complementary domain of a continuous curve is the sum of three mutually exclusive point-sets $S_{1}^{\prime}, S_{2}$ and $[P]$, where (1) $S_{1}^{\prime}$ is a countable set of simple closed curves no two of which have more than one point in common and whose interiors have no point in common (unless one of these simple closed curves be the outer boundary in the case of a bounded complementary domain), (2) $S_{2}$ is a countable set of simple continuous arcs no two of which have in common an interior point of both, and (3) $[P]$ is a totally disconnected set of limit points of the set $S_{1}^{\prime}+S_{2}$.

Proof. (1) has been established in Theorem 4.

Let $\beta$ be the boundary of a complementary domain of a continuous curve, and $P$ a point of $\beta-S_{1}^{\prime \prime}$. That maximal connected subset of $\beta-S_{1}^{\prime}$ determined by $R$ I shall call a set of type $Q$, provided it consists of more than one point. A set of type $Q$, together with its limit points, I shall call a set of type $N$.

By Theorem 11, a set of type $N$ is a continuous curve. Furthermore, it is a continuous curve that contains no simple closed curve. For, suppose $N$ is a set of type $N$ that contains a simple closed curve $C$. Then

and as

$$
S_{1}^{\prime} \supset C \text {. }
$$

$$
\beta-S_{1}^{\prime} \supset Q
$$

where $Q$ represents that set of type $Q \cdot$ which determines $N$, then must

$$
N-Q \supset C
$$


That is, every point of $C$ is a limit point of $Q$ that does not belong to $Q$.

Let $d$ be that maximal domain with respect to $\beta$ which is a subset of $\beta-C^{\gamma}$ and of which $Q$ is a subset. Then every point of $C$ is a boundary point of $d$ with respect to $\beta$. Clearly this is a contradiction of Theorem 16. Hence no set of type $N$ can contain a simple closed curve.

Applying Theorem 15, every set of type $N$ is the sum of a countable set of arcs, no two of which have in common an interior point of both, together with a totally disconnected set of limit points of these arcs. All arcs of $\beta$ so determined assign to a set $S_{2}$.

The sets $S_{1}^{\prime}$ and $S_{2}$ are mutaally exclusive. For, suppose they have in common a point $x$. Then $x$ belongs to some arc, $a$, of some set $N$ of type $N$, and, as determined, no points of $a$ are end-points of $N$ (see proof of Theorem 15). It follows, that $x$ is a cut-point of $N$. Hence $N-x$ is the sum of two mutually separated point-sets, $N_{1}$ and $N_{2}$. Both $N_{1}$ and $N_{2}$ must contain points of $Q$, the set of type $Q$ which determines $N$. But this is impossible, since $Q$ is a connected subset of $\beta-S_{1}^{\prime}$, and hence of $\beta-x$. It follows that the sets $S_{1}^{\prime}$ and $S_{2}$ are mutually exclusive, and it also follows that every arc of $S_{2}$ is a subset of some set of type $Q$.

If $a$ and $b$ are two arcs of $S_{2}$, then (1) if $a$ and $b$ belong to the same set of type $Q$, they have in common no point which is an interior point of both, and (2) if $a$ and $b$ do not belong to the same set of type $Q$ they have no points in common. It follows that the arcs of $S_{2}$ are countable (as a consequence of Theorem 5).

The set of points

$$
[P]=\beta-\left(S_{1}^{\prime}+S_{2}\right)
$$

is a totally disconnected set of points. For, if there exists a connected subset, $t$, of $[P]$, then $t$ is a subset of some set of type $Q$, as

$$
\beta-S_{1}^{\prime} \supset t \text {. }
$$

If $N$ is the set of type $N$ determined by this set of type $Q$, and $[a]$ the set of ares common to $N$ and $S_{2}$, then $N-[a]$ is totally disconnected by Theorem 15. But

$$
N-[a] \supset t \text {. }
$$

It follows that $t$ cannot be a connected set, and that $[P]$ is totally disconnected.

This completes the proof of the Theorem. 
III. Characterizations of continuous curves, and of the boundaries of the complementary domains of plane continnous curves ${ }^{1}$ ).

In this section I shall establish a condition which continua that are not connected im kleinen must satisfy. By means of this condition I shall characterize continuous curves for any number of dimensions, and the boundaries of the complementary domains of plane continuous curres.

Lemma I. If a bounded continuum $M$ is not a continuous curve, then there exist two concentric circles $K_{1}$ and $K_{2}$, and a sequence of sub-continua of $M$.

$$
M_{\infty}, M_{1}, M_{2}, M_{3}, \ldots
$$

such that (1) each of these sub-continua contains at least one point on $K_{1}$ and $K_{2}$, respectively, but no points exterior to $K_{1}$ or interior to $K_{2}$, (2) no two of these sub-continua have a point in common, and no two of them contain points of any connected subset of $M$ which lies wholly in the set $K_{1}+K_{2}+I$, (where $I$ is the annular domain bounded by $K_{1}$ and $K_{2}$ ), (3) $M_{\infty}$ is the sequential limiting set of the sequence $M_{1}, M_{2}, M_{3}, \ldots$, (4) if $K$ is that maximal sub-continuum of $M$ containing $M_{\infty}$ and lying wholly in the set $K_{1}+K_{2}+I$, then all of the continua $M_{1}, M_{2}, M_{3}, \ldots$, lie in a connected subset of $M-K$.

Proof: R. L. Moore has established conditions (1), (2) and (3) of this Lemma 2). It remains to establish condition (4).

$R_{K_{i}}$ being the radius of the circle $K_{i}$, Let $K_{3}$ and $K_{4}$ be two circles concentric with $K_{1}$, and such that

$$
R_{R_{1}}>R_{E_{3}}>R_{E_{4}}>R_{R_{2}} \text {. }
$$

For every value of $n,(n=\infty, 1,2,3, \ldots)$ there exists a continuum $M_{n}^{*}$ such that

$$
M_{n} \supset M_{n}^{*}
$$

and such that if in the statement of this Lemma each $M_{i}$ is replaced by $M_{i}^{*}, K_{1}$ and $K_{2}$ replaced by $K_{3}$ and $K_{4}$, respectively, and $K$ replaced by $T$, statements (1), (2), and (3) hold true, but (4)

1) The theorems and lemmas in this section were presented to the American Mathematical Society, Dec. 29, 1922.

2) A characterization of Jordan regions by properties having no reference to their boundaries, loc. cit. 
may or may not hold true. If statement (4) does hold true, the proof is complete. If statement (4) does not hold true, let $K_{5}$ and $K_{6}$ be circles concentric with $K_{1}$ and such that

$$
R_{\bar{K}_{4}}>R_{E_{6}}>R_{K_{6}}>R_{E_{2}} \text {. }
$$

For every value of $n,(n=\infty, 1,2,3, \ldots)$, there exists a continuum $\bar{M}_{n}$ such that

$$
M_{n} \supset \bar{M}_{n}
$$

and such that if in the statement of this Lemma each $M_{i}$ is replaced by $\bar{M}_{i}, K_{1}$ and $K_{2}$ replaced by $K_{5}$ and $K_{6}$, respectively, and $K$ replaced by $W$, statements (1), (2), and (3) hold true, but (4) may or may not hold true. I shall prove that (4) must hold true. There exists an infinite sequence of distinct sets, $L_{1}, L_{2}, L_{8}, \ldots$ such that (1) for each $n . L_{n}$ is a maximal connected subset of $M-T$, and contains at least one set, but at most a finite number of sets of the sequence $M_{1}, M_{2}, M_{3}, \ldots$, (2) every set of the sequence $M_{1}$, $M_{2}, M_{3}, \ldots$ belongs to some set of the sequence $L_{1}, L_{2} . L_{3}, \ldots$

For every value of $n,(n=1,2,3, \ldots), L_{n}$ has a limit point in $T$. For, suppose $L_{i}$ is a set of this sequence that has no limit point in $T$. Then, since $L_{i}$ is a maximal connected subset of $M-T$, $L_{i}$ is closed. $L_{i}$ and $T$ are then two mutually separated continua.

There exists a connected domain $D_{1}$ containing $L_{i}$, but containing no point of $T$ nor having a point of $T$ on its boundary.

If $A$ is a point of $L_{i}$ and $B$ a point of $T$, then $M$ is a continuum containing $A$ and $B$. That is, $M$ contains a point $A$ interior to $D_{1}$ and a point $B$ exterior to $D_{1}$. Then there exists a sub continuum $Q$ of $M$, which contains $A$ and at least one point $x$ on the boundary of $D_{1}$, but no point exterior to $D_{1}{ }^{1}$ ).

$x$ cannot belong to $T$ and hence belongs to $M-T$; furthermore $x$ is joined to $A$ by a subset $Q$ of $M-T$, and must therefore belong to $L_{i}$. But no points of $L_{i}$ lie on the boundary of $D_{1}$. Therefore the supposition that $L_{\mathbf{b}}$ has no limit point in $T$ leads to a contradiction. Hence every set $L_{n}(n=1,2,3, \ldots)$ has a limit point in $T$.

Not more than one set of the sequence $L_{1}, L_{2}, L_{3}, \ldots$ has a li-

1) Cf. Anna M. Mnlliken, Certain theorems relating to plane connected pointsets, Trans. Amer. Math. Soc., XXIV (1923) Th. 1. 
mit point in $W$. For if one of these sets, say $L_{j}$, has a limit point in $W$, then must

$$
L_{j} \supset W
$$

and no two of these sets have a point in common. We can consider $L_{j}$ as being omitted from the sequence $L_{1}, L_{2}, L_{3}, \ldots$ We have, then, an infinite sequence of connected sets, $L_{1}, L_{\mathbf{2}}, L_{\mathbf{3}}, \ldots$ such that for every $n(n=1,2,3, \ldots)$,

$$
M-W \supset L_{n}
$$

and $L_{n}$ has a limit point in $T$. Since $T$ is connected, the set

$$
U=L_{1}+L_{2}+L_{3}+\ldots+T
$$

is a connected subset of $M-W$. Since every set of the sequence $\bar{M}_{1}, \bar{M}_{2}, \bar{M}_{3}, \ldots$ belongs to some set of the sequence $L_{1}, L_{2}, L_{3}, \ldots$, and therefore to $U$, condition (4) of the theorem is satisfied by the sequence $W, \bar{M}_{1}, \bar{M}_{2}, \bar{M}_{3}, \ldots$ as also are conditions (1), (2), and (3), if, as already pointed out, $K$ is replaced by $W$, each $M_{i}$ by $\bar{M}_{i}$, and $K_{1}$ and $K_{2}$ by $K_{5}$ and $K_{6}$, respectively.

Although the above proof is given for two dimensions, it should be observed that a similar proof can be given to show that the Lemma holds for any number of dimensions.

Lemma 2. If $x$ and $y$ are two points of the boundary, $\beta$, of a complementary domain of a continuous curve, there exist at most two distinct arcs from $x$ to $y$; $i$. e., arcs that have in common at most their end-points, $x$ and $y$, and if two such arcs exist every arc from $x$ to $y$ which belongs to $\beta$ is identical with one of these arcs.

(This Lemma is a corollary of Theorem 4).

It has recently been shown by R. L. Moore that every two points that lie together in a connected subset of an open ${ }^{1}$ ) subset of a continuous curve, lie in a sub-continuum of that open subset ${ }^{2}$ ). Using Lemma 1, it is easy to show that any bounded coutinuum which has this property is a continuous curve.

Theorem 18. In order that a bounded continuum $M$ should be a continuous curve, it is necessary and sufficient that every two points that lie in a connected subset of an open subset of $M$ lie in a subcontinuum of that open subset.

1) If $L$ is a closed proper subset of a continuum, $M$, then $M-L$ is an open subset of $M$.

2) R. L. Moore, Concerning continuous curves in the plane, loc. cit. Theorem 1. 
[The proof of this theorem for the two-dimensional case is given below. An analogous proof may be given to show that the theorem holds in $m$ dimensions]

(1) The condition stated in the theorem is necessary, as shown by R. L. Moore.

(2) The condition stated in the theorem is sufficient. For, suppose $M$ is a bounded continuum satisfying the condition stated in the theorem, but which is not a continuous curve. Then the conditions of Lemma 1 are satisfied.

Let $A$ and $B$ be two points of $M_{\infty}$ that lie in $I$, and let $C_{1}$ and $C_{2}$ be two circles with centers $A$ and $B$, respectively, such that (i) $C_{1}$ and $C_{2}$ have no point in common nor have their interiors any point in common, and (ii) $C_{1}$ and $C_{2}$ lie wholly in $I$, but do not enclose $K_{2}$.

If $Q_{1}$ an $Q_{2}$ denote the sets of all points of $K$ interior to $C_{1}$ and $C_{2}$, respectively, let

$$
T=K-\left(Q_{1}+Q_{2}\right)
$$

$T$ is closed, and therefore $M-T$ is an open subset of $M$. Since

$$
K \supset T
$$

all of the continua $M_{1}, M_{2}, M_{3}, \ldots$ lie in a connected subset. $U$, of $M-T$.

Since $A$ and $B$ belong to $M_{\infty}$, they are limit points of the sequence $M_{1}, M_{2}, M_{3}, \ldots$ and therefore limit points of $U$. The set $U+A+B$ is therefore a connected subset of $M-T$.

By hypotbesis every two points that lie in a connected subset of an open subset of $M$ lie in a sub-continuum of that open subset. Then $A$ and $B$ must lie in a continuum $N$, such that

$$
M-T \supset N \text {. }
$$

Since $N$ is a continuum containing a point $A$ interior to $C_{1}$ and a point $B$ exterior to $C_{1}$, there exists a sub-continuum $N_{1}$ of $N$ which contains $A$ and at least one point $P$ on $C_{1}$, but no puint exterior to $C_{1}$. Since $C_{1}$ lies wholly in $I$, and does not enclose $K_{2}$, $P$ is joined to $A$ by a continuum of $M$ that lies in $I$ and must therefore be a point of the set $K$, but not of the set $Q_{1}$ or the set $Q_{2}$. That is. $P$ must be a point of the set $T$. But $P$ also is a point of the $\operatorname{set}^{i} N$, which is a subset of the set $M-T$. Hence the 
supposition that $M$ is not a continuous curve has led to a contradiction, and $M$ must therefore be a continuous curve.

Theorem 19. In order that the boundary of a simply connected domain should be a continuous curve, it is necessary and sufficient that every connected subset of it be connected in the strong sense ${ }^{1}$ ).

$A$. The condition stated in the theorem is necessary. Let $M$ be a continuous curve which is the boundary of a simply connected domain $D$, and let $N$ be any connected subset of $M$. I shall show that any two points $x$ and $y$ of $N$ are the extremities of an arc that lies wholly in $N$. There are three cases to consider:

(1) $x$ and $y$ the extremities of two distinct (in the sense defined in Lemma 2) arcs, $a_{1}$ and $a_{2}$, of $\boldsymbol{M}$.

If $N$ contains no arc from $x$ to $y$, there exists at least one point, $P_{1}$, on $a_{1}$, and at least one point, $P_{2}$, on $a_{2}$, such that neither $P_{1}$ nor $P_{2}$ belongs to $N$.

Add to $N$ its limit points, and call the resulting set $N^{\prime}$. By virtue of Theorem 11, $N^{\prime}$ is a coutinuous curve, and hence contains an arc, $a$, from $x$ to $y$. As a result of Lemma 2 either $a \equiv a_{1}$, or $a \equiv a_{2}$. Suppose that $a \equiv a_{1}$. Then $P_{1}$ belongs to $N^{\prime}$, but not to $N$. But the set $N^{\prime}-P_{1}$ must then contain a connected subset $N$, containing $x$ and $y$, and therefore an are from $x$ to $y^{2}$ ). By Lemma 2 this arc must be the are $a_{2}$. However, the set $N^{\prime}-\left(P_{1}+P_{2}\right)$ also contains a connected subset, $N$, containing $x$ and $y$, and hence an are from $x$ to $y$; call this arc $a_{3}$. We have, then, two distinct arcs, $a_{1}$ and $a_{2}$, from $x$ to $y$, and a third are, $a_{3}$, which is identical with neither $a_{1}$ nor $a_{2}$. But this is a contradiction of Lemma 2. In this case, then, $N$ must contain an arc from $x$ to $y$.

(2) $x$ and $y$ the extremities of only one are, $a$. of $M$.

In this case, if $P$ is a point of $a$ not belonging to $N$, the set $N^{\prime}-P$ contains an are from $x$ to $y$ which cannot be identical with $a$, thus giving an immediate contradiction of the hypothesis that $M$ contains only one arc from $x$ to $y$. In this case also, then, must $N$ contain an are from $x$ to. $y$.

(3) $x$ and $y$ neither the extremities of two distinct ares of $M$, nor of only one are of $M$, but the extremities of a totality of arcs, $T$, such that if $a_{1}$ and $a_{2}$ are any two $\operatorname{arcs}$ of $T, a_{1}$ and $a_{2}$ have

1) A point-set $M$ is said to be connected in the strong sense if overy two points that lie in a connected subset $N$ of $M$ lie also in a sub-continuam of $N$.

2) Cf. R. L. Moore, Concerning continuous curves in the plane, loc. cit.; Th. 1. 
interior points in common, and each has interior points that do not belong to the other.

Let $F$ denote a set of points such that (1) if $P$ is any point of $F$, $P$ lies on every are of $T$, and (2) if $P$ is a point that lies on every arc of $T, P$ belongs to $F$. That such points exist, distinct from $x$ and $y$, can be easily shown as follows: Let $a_{1}$ be any arc of $T$. Suppose $a_{2}$ is any other are of $T$, and let $P$ be a point of $a_{2}$ not on $a_{1} . P$ is an interior point of an are $a_{2}^{\prime}$ which is a subset of $a_{2}$, and which has in common with $a_{1}$ only its end-points; call these end-points $A$ and $B$. Either $A$ is distinct from $x$ and $y$ or $B$ is distinct from $x$ and $y$. Let $A$ be distinct from $x$ and $y$. Then $A$ is a point of $F$. For, suppose $A$ is not a point of $F$. Then some arc, $a_{3}$, of $T$, does not contain $A$. Let $A^{\prime}$ be the last point of $a_{3}$ on the arc $x A$ of $a_{1}$ in the order from $x$ to $A$; on the arc $A^{\prime} y$ of $a_{3}$, in the order from $A^{\prime}$ to $y$, let $B^{\prime}$ be the first point that $a_{3}$ has in common with the point-set consisting of the arc $A y$ of $a_{1}$ and the arc $a_{2}^{\prime}$. That arc of $a_{3}$ from $A^{\prime}$ to $B^{\prime}$ call $a_{3}^{\prime}$. The set $a_{1}+a_{2}^{\prime}+a_{3}^{\prime}$ contains two points that are the end-points of three distinct arcs, a contradiction of Lemma 2. Therefore $A$ must be a point of $F$ dislinct from $x$ and $y$. Similarly, all points common to $a_{1}$ and $a_{2}$ are common to all arcs of $T$ and hence belong to $F$. Either $N$ contains all points of $F$ or it does not.

Suppose $N$ contains. all points of $F$. If $P$ is any point of $a_{1}$ that is not a point of $F$, let $a_{2}$ be an arc of $T$ that does not contain it. In the order from $x$ to $y$ let $x^{\prime}$ be the last point $a_{2}$ has in common with the arc $x P$ of $a_{1}$, and $y^{\prime}$ the first point after $x^{\prime}$ that $a_{2}$ has in common with the arc $P y$ of $a_{1}$. Since, as shown above, $x^{\prime}$ and $y^{\prime}$ are points of $F$, they belong to $N$, and as they are the end-points of two distinet ares of $M$, the proof of case (1) of this theorem shows that $N$ contains one of these arcs. Similarly, every point $P$ of $a_{1}$ that does not belong to $F$ determines two points $x^{\prime}$ and $y^{\prime}$ of $F$ which are the end-points of an arc that belongs to $N$. Let $\bar{L}$ dencte the point-set obtained by adding together the points of all the ares of the set $L$.

The point-set $\bar{L}+F$ contains an are $t$ from $x$ to $y$. This fact can be established by showing that $\bar{L}+F$ is a continuous curve. $\bar{L}+F$ is a closed set. For, (i) $F$ is closed. If $P$ is a limit of $F$, then, since $F$ is a subset of $a_{1}$, and $a_{1}$ is closed, $P$ must belong to $a_{1}$. If $a_{2}$ is any otber arc of $T, P$ lies on $a_{2}$ for the same rea- 
son. That is, $P$ is common to all ares of $T$, and hence belongs to $F$. Also, (ii) all limit points of $\bar{L}$, if they do not belong to $\bar{L}$, belong to $F$. For, let $P$ be a limit point of $\bar{L}$ that does not belong to $\bar{L}$. Let $k$ be a circle of arbitrary radius $r$ and center $P$, and $k^{\prime}$ a circle of radius $r / 2$, concentric with $k . k^{\prime}$ must contain points of an infinite set of ares of $L$. But since $L$ is a subset of $\boldsymbol{M}$ and $M$ cannot contain an infinite set of arcs of diameter greater than $r / 2$ that have only end.points in common (Theorem 6) then must an infinite set of ares of $L$ lie wholly interior to $k$. But the end-points of each such arc of $L$ must then lie interior to $k$, and as the radius of $k$ is arbitrary it follows that $P$ is a limit point of such end-points; i. e., of $F$. Therefore if $P$ is not a point of $\bar{L}$ it is a point of $F$. Since $F$ is closed, and $\bar{L}$ is either closed or those limit points that it does not contain belong to $F$, it follows that $F+\bar{L}$ is closed. As $\bar{L}+F$ is connected and a subset of $M$, $\bar{L}+F$ is a bounded continuum, and by virtue of Theorem 16 a continuous curve, therefore containing an arc from $x$ to $y$. As $\bar{L}+F$ is a subset of $N$, it follows that $N$ contains an are from $x$ to $y$.

Suppose $N$ does not contain all points of $F$; that is, that there exists some point $P$. of $F$ that $N$ does not contain. If, as in case (1) of this theorem, $N^{\prime}$ denotes the set $N$ together with its limit points, then $N^{\prime}$ is a continoous curve and therefore contains an arc from $x$ to $y$; this arc must belong to $T$, and hence contains $P$. That is, $P$ belongs to $N^{\prime}$, but not to $N$. Then $N^{\prime}-P$ contains a connected subset, $N$, containing $x$ and $y$, and as shown in case (1) of this theorem must therefore contain an arc from $x$ to $y$ that does not contain $P$. But this is impossible, since $P$ is a point of $F$ and therefore common to all arcs from $x$ to $y$. Hence $N$ must contain all points of $F$, and therefore, as shown above, an are from $x$ to $y$.

$B$. The condition is sufficient by Theorem 18 .

It will be noted that the above proof also establishes the following theorem:

Theorem 20. In order that the boundary of a simply connected domain should be a continuous curve, it is necessary and sufficient that every connected subset of it be arc-wise ${ }^{\mathrm{I}}$ ) connected.

1) A point-set $M$ is said to be arc-wise connected when every two points of $M$ are the extremities of a simple continuous arc that lies wholly in $M$. 